\title{
Reshaping diagrams for bending straightening of forged aeronautical components
}

\author{
Ramiro Mena ${ }^{1,2,3}$. José V. Aguado ${ }^{2}$ - Stéphane Guinard ${ }^{1}$ - Antonio \\ Huerta $^{3,4}$
}

Received: date / Accepted: date

\begin{abstract}
Large and thick-walled aluminium forgings exhibit shape distortions induced by residual stresses. To restore the nominal geometry, a series of highlymanual and time-consuming reshaping operations need to be carried out. In this paper, we are concerned with the development of efficient computer simulation tools to assist operators in bending straightening, which is one of the most common reshaping operations. Our approach is based on the computation of reshaping diagrams, a tool that allows selecting a nearly optimal bending load to be applied in order to minimize distortion. Most importantly, we show that the reshaping diagram needs not to account for the residual stress field, as its only effect is to shift of the reshaping diagram by some offset. That is, the overall behaviour including a realistic 3D residual stress field in a forged
\end{abstract}

$\triangle$ Ramiro Mena

ramiro-francisco.mena-andrade@airbus.com ramiro-francisco.mena-andrade.2@ec-nantes.fr

$\triangle$ José V. Aguado

jose.aguado-lopez@ec-nantes.fr

$\triangle$ Stéphane Guinard stephane.guinard@airbus.com

$\triangle$ Antonio Huerta rantonio.huerta@upc.edu

1 Airbus SAS, 18 rue Marius Terce, 31300 Toulouse, France.

2 Institut de Calcul Intensif (ICI) at Ecole Centrale de Nantes, 1 rue de la Noë, 44300 Nantes, France.

3 Laboratori de Càlcul Numèric (LaCàN). E.T.S. de Ingenieros de Caminos, Canales y Puertos, Universitat Politecnica de Catalunya, Barcelona, Spain.

4 Centre Internacional de Mètodes Numèrics a l'Enginyeria (CIMNE), Barcelona, Spain part can be retrieved by shifting the residual stress free reshaping diagram by the appropriate offset. Finally, we propose a strategy in order to identify the offset on-the-fly during the reshaping operation using simple force-displacement measures.

Keywords Residual Stresses - Shape distortion · Bending straightening · Forged parts · Numerical simulation · Reshaping diagrams

\section{Introduction}

Large and thick-walled aluminium forgings are widely used as structural parts in the aeronautical industry. The good formability of aluminium, together with its great strength-to-weight ratio, allows producing complex forged parts in an economical way [?]. Additionally, when compared to other metal working processes, such as extensive machining, welding or casting, improved material properties in terms of grain size and orientation are obtained [?].

Aluminium forgings undergo a multiple-step manufacturing process to produce the final aeronautical component. We describe here a typical manufacturing sequence, although others are of course possible. First, forged blanks are typically produced on hydraulic presses with hot closed-dies [?]. The process continues with a solution heat treatment (SHT) followed by quenching, which is required to improve the mechanical properties of the material. As a counterpart, the quenching step is the main responsible for the creation of residual stresses due to the strong thermal gradients between the surface and the core of the part. Bi-axial compression stress state develops at the surface, counterbalanced by a three-dimensional tension state in the core [?]. After 


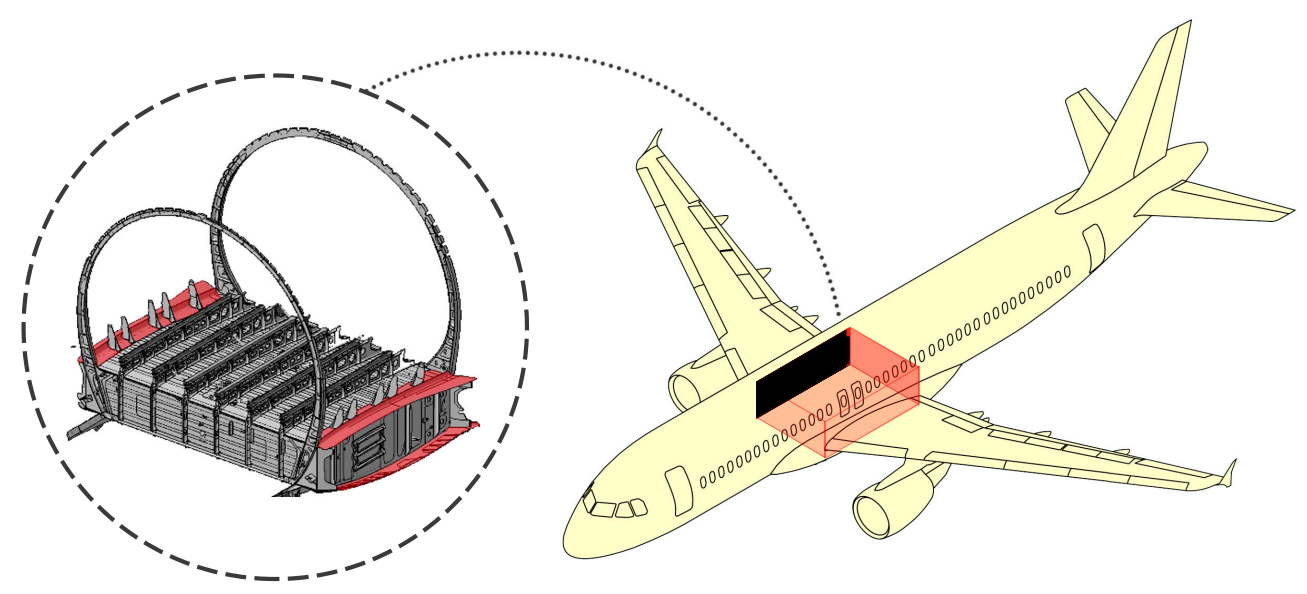

Fig. 1: Example of aluminium forgings subjected to distortion: cruciform beams of the Airbus' A320 wing box, highlighted in red.
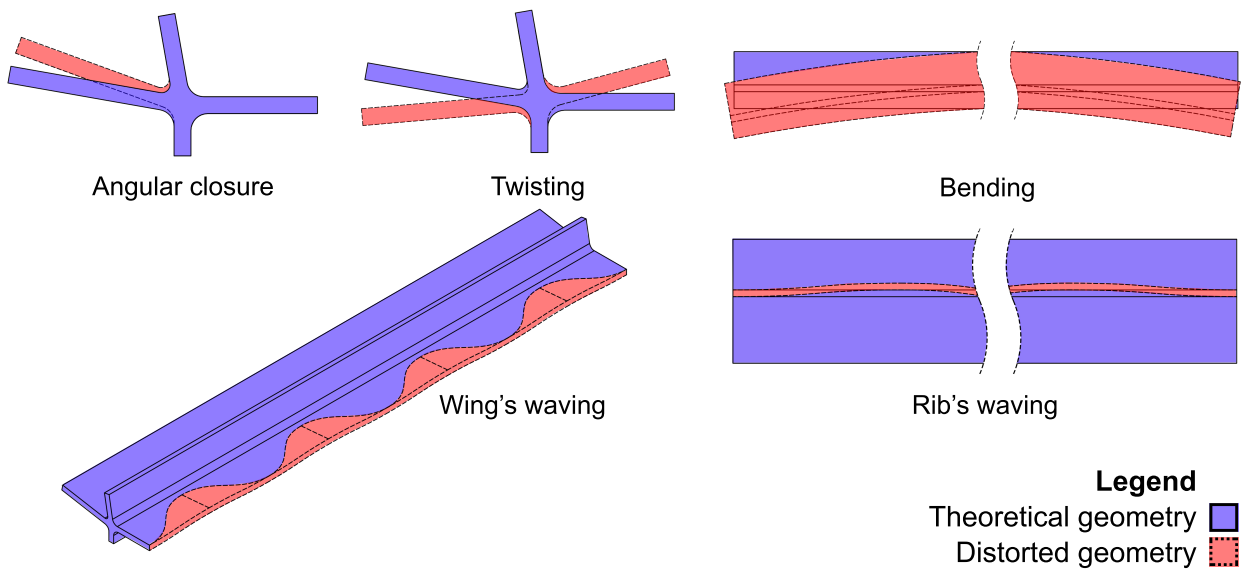

Fig. 2: Principal types of distortion after machining in a simplified version of the Airbus' A320 cruciform beam. Adapted from [?].

quenching, the components usually undergo a stress relief process (e.g. compression), followed by an ageing treatment, to reach the peak yield stress. Then, machining is used to obtain the final geometry from the blank. During this step, material removal breaks the stress equilibrium, producing a redistribution of residual stresses (RS) and, consequently, shape distortions $[?, ?]$.

Distortions exhibit a strong variability, changing from part to part, therefore being hard to anticipate. As a practical solution, the manufacturing process incorporates a post-machining step called reshaping, which consists in a sequence of mechanical operations to reestablish the nominal geometry of the part. The idea is to produce localized plastic strains in specific areas with the aim to counteract distortion. Besides geometrical constraints, it is worth to recall that the repaired part must also comply with the guidelines imposed by the engineering office, to ensure that its mechanical state is compatible with its operational role. Due to the strong distortion variability, reshaping turns out to be a collaborative, highly manual process, as multiple operators may be involved to repair one single part. It depends heavily on the experience of the operators, and repairing each structural part is very time-consuming. Some studies suggest that the impact of reshaping can be as high as $50 \%$ of the final cost of the component, thus compromising any manufacturing profitability [?]. Hence, there is a strong demand from the industry to derive operational assistance from numerical simulation tools [?].

Cruciform beams, located at the wing box of the aircraft (see Figure ??) are an example of forged structural component subjected to reshaping. The wing box is often referred to as the structural heart of the aircraft, as it connects the wings to the fuselage and bears heavy 
loads [?]. Being part of the wing box, cruciform beams must meet tight dimensional tolerances to facilitate the assembly process and to avoid undesired pre-loads in such an important part of the aircraft.

Forged parts such as cruciform beams tend to develop complex RS states, leading to complex distortion patterns. Marin [?] documented the principal types of distortions present on a cruciform as shown in Figure ??, where each type of distortion is represented independently (although most often a combination of them is observed).

Several reshaping operations can be found in the literature to repair a distorted part. Among them, we can cite the following: bending straightening [?,?], torsion straightening [?,?], roller burnishing [?,?] and ultrasonic needle peening [?,?]. While bending and torsion create non-homogeneous deformation acting along the whole cross-section, burnishing and peening induce local compressive residual stresses only at the surface level. In this paper, we focus on the bending straightening operation only, which essentially consists in applying a load in the opposite direction to observed distortions. Bending straightening is an iterative loadingunloading process, where, for a given initial distortion, the operator has to guess: i) where to place the supports; ii) where to apply the bending load; and iii) how much loading needs to be applied to minimize distortion.

Diverse authors have proposed to use numerical simulation as a means to anticipate the resulting shape after a correction step. The idea is that, from the knowledge of the full residual state field prior to reshaping, it should be possible to simulate the correction steps. As residual stresses cannot be systematically measured in an industrial context, lots of efforts have been devoted to derive numerical models that are able to estimate them $[?, ?]$. These models require simulating, either wholly or in part, the multi-step manufacturing process. We shall refer to this approach throughout the paper as the sequential approach, as depicted in Figure ??. Although the sequential approach can provide insightful results when good knowledge on both process conditions and material behaviour are available, most often, significant mismatch between numerical predictions and experimental observations is reported, e.g. [?]. Assessing how uncertainties propagate through the different manufacturing steps (i.e. from quenching to machining) is key to keep a high confidence level on the predictions from sequential models [?,?].

In this work, we deviate from the sequential approach and propose an alternative route to simulate bending straightening of forged components. We first introduce a simple tool, the reshaping diagram, which provides the remaining distortion (i.e. after unloading) as a function of the applied bending load (for fixed rollers position and residual stress field). Note that the reshaping diagram is a very convenient tool to assist the operator: once it is made available, the reshaping operation reduces to select the optimal bending load that minimizes the remaining distortion. Unfortunately, computing the reshaping diagram requires a precise knowledge on the residual stress field, which as discussed above, is not a trivial task. To overcome this issue, we propose a workaround that uses distortion (which is measurable) as the main input, instead of residual stresses. The underlying idea is that to minimize distortion we may not need a precise knowledge of the residual stress field, but only its influence on the reshaping diagram. Therefore, we compute the reshaping diagram of a distorted part without residual stresses, that is, we keep the distorted geometry after machining but suppress the residual stress field. We shall refer to this reshaping diagram throughout the entire paper as the residual stress free (RSF) approach (see Figure ??). Then, we show that the effect of the RSF hypothesis results only in a shift of the reshaping diagram. That is, the overall behaviour including a realistic 3D residual stress field in a forged part can be retrieved by shifting the original reshaping diagram by the appropriate offset. Finally, we propose a strategy in order to identify the offset on-the-fly during the reshaping operation using simple force-displacement measures. One of the main advantages of the RSF approach is that it does not require any prior knowledge on the residual stress field; it only requires information on the distorted shape, which unlike the residual stresses, can be systematically measured in an industrial environment.

The rest of the paper is organized as follows. In section ??, we present numerical models for residual stress prediction and bending straightening simulation, and validate them against available data in the literature. Using the validated models, in section ?? we create realistic 3D stress states for forged parts and compute the reference reshaping diagram by simulating the bending straightening process. The influence of various process parameters is also analysed. In section ??, we repeat the operation with the residual stress free hypothesis and observe that the obtained RSF reshaping diagram differs only by some offset from the reference one. In addition, we derive a simple approach to calibrate the offset on-the-fly during the reshaping operation using simple force-displacement measures. Finally, in section ??, we draw some conclusions and perspectives for future work. 


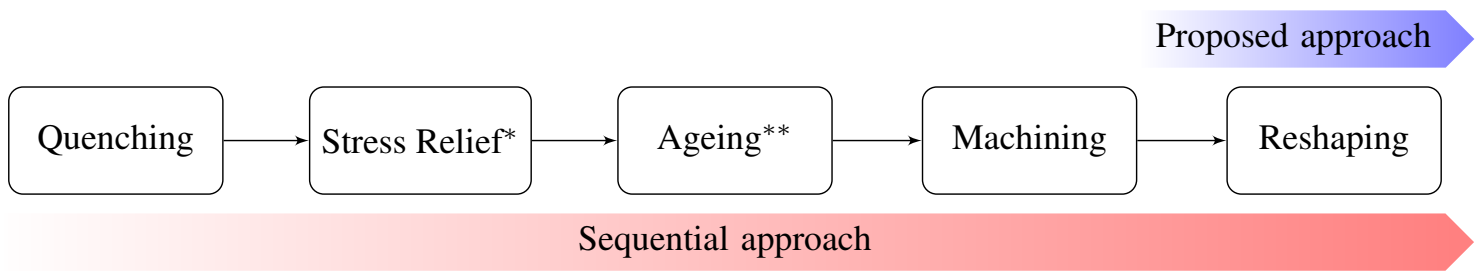

Fig. 3: Reshaping simulation: sequential vs proposed approach ( ${ }^{*}$ not included and $*^{*}$ included but not simulated).

\section{Numerical models description and validation}

In this section we present and validate numerical models for residual stress prediction and bending straightening. While aluminium forgings present a complex threedimensional residual stress state [?,?,?], the residual stresses are well known for rolled plates and dominated by the longitudinal stresses within the thickness $[?, ?, ?]$. Experimental data on forged parts is scarce, given the difficulty of measuring complex 3D residual stress fields. Therefore, the numerical models presented in this section are validated against data for rolled plates, and then used in subsequent sections as a means to produce reference results in forged parts.

For the implementation, we rely on a well-known and extensively-used software package, Abaqus [?], based on the finite element method. We therefore provide a synthetic description of the models, making use of summarizing tables, and put more emphasis on the validation part.

\subsection{Quenching simulation for residual stress prediction}

The residual stresses generated after the heat treatment of quenching can be simulated as a sequentially coupled thermo-plasticity problem [?,?]. The problem is divided into two computational steps: the heat transfer step and the mechanical step. For the heat transfer step, the heat conduction equation is solved first. Once the temperature evolution in time is known, it is used as an input for the mechanical problem, which is affected via thermal expansion and the temperature-dependent constitutive model. By solving the mechanical step, the residual stress state is computed.

\subsubsection{Thermo-plasticity modelling}

The thermo-plasticity model used for quenching simulation is synthetically presented in Table ??. To this end, we consider a body that occupies an open bounded domain $\Omega \subset \mathbb{R}^{d \leq 3}$. Its boundary is denoted by $\partial \Omega$. We suppose that the part is at temperature $T_{0}$ (temperature of the solution heat treatment) at an initial time $t=0$, when it is submerged into a liquid at a predefined temperature $T_{\infty} \ll T_{0}$ for a fast cooling during a time interval of interest $t \in\left[0, T_{\text {final }}\right]$. Heat transfer from the part to the surrounding liquid through the part's boundary $\partial \Omega$ is modelled as a convective boundary condition with a heat transfer coefficient denoted by $h$. By $T:=T(\boldsymbol{x}, t)$, we denote the temperature evolution at a point $\boldsymbol{x} \in \Omega$ and time $t \in\left[0, T_{\text {final }}\right]$.

Once the heat transfer step is solved, the resulting temperature field is introduced in the mechanical step as a predefined field. As a consequence, volumetric strains are generated by the effect of thermal expansion. The strain tensor $\varepsilon$ decomposed as the sum of an elastic component $\varepsilon^{e}$, a plastic component $\varepsilon^{p}$ and a thermal component $\varepsilon^{t h}$. Classical $J_{2}$ plasticity with RambergOsgood [?] isotropic hardening is implemented.

\subsubsection{Application to a rolled plate}

In order to validate the model for residual stress prediction, we simulate a case from the literature for which residual stresses are available, see [?]. Specifically, we apply the model described in section ?? to a rolled plate data of $760 \times 760 \times 77.9 \mathrm{~mm}$. The plate is oriented such that the $x, y$ and $z$ axis are parallel to the longitudinal (L), longitudinal-transverse (LT) and short transverse (ST) directions, respectively. Based on the symmetry present in all directions, only $1 / 8$ of the geometry is simulated.

The quenching process conditions are summarized in Table ??. The rest of the modelling parameters given in Table ?? (i.e. $\rho, C_{p}, \kappa, h, \alpha, E, \nu, \sigma_{y 0}, k$ and $n$ ) were taken from [?]. As most of them are temperaturedependent, we do not report them here for the sake of brevity.

Concerning the numerical model, the plate was meshed with 64,000 8-node linear hexahedron elements, with 40 elements in the through-thickness (ST) direction. The total number of nodes was 68,921. Abaqus element types are DC3D8 for the heat transfer analysis 
Table 1: Thermo-plasticity model for quenching simulation.

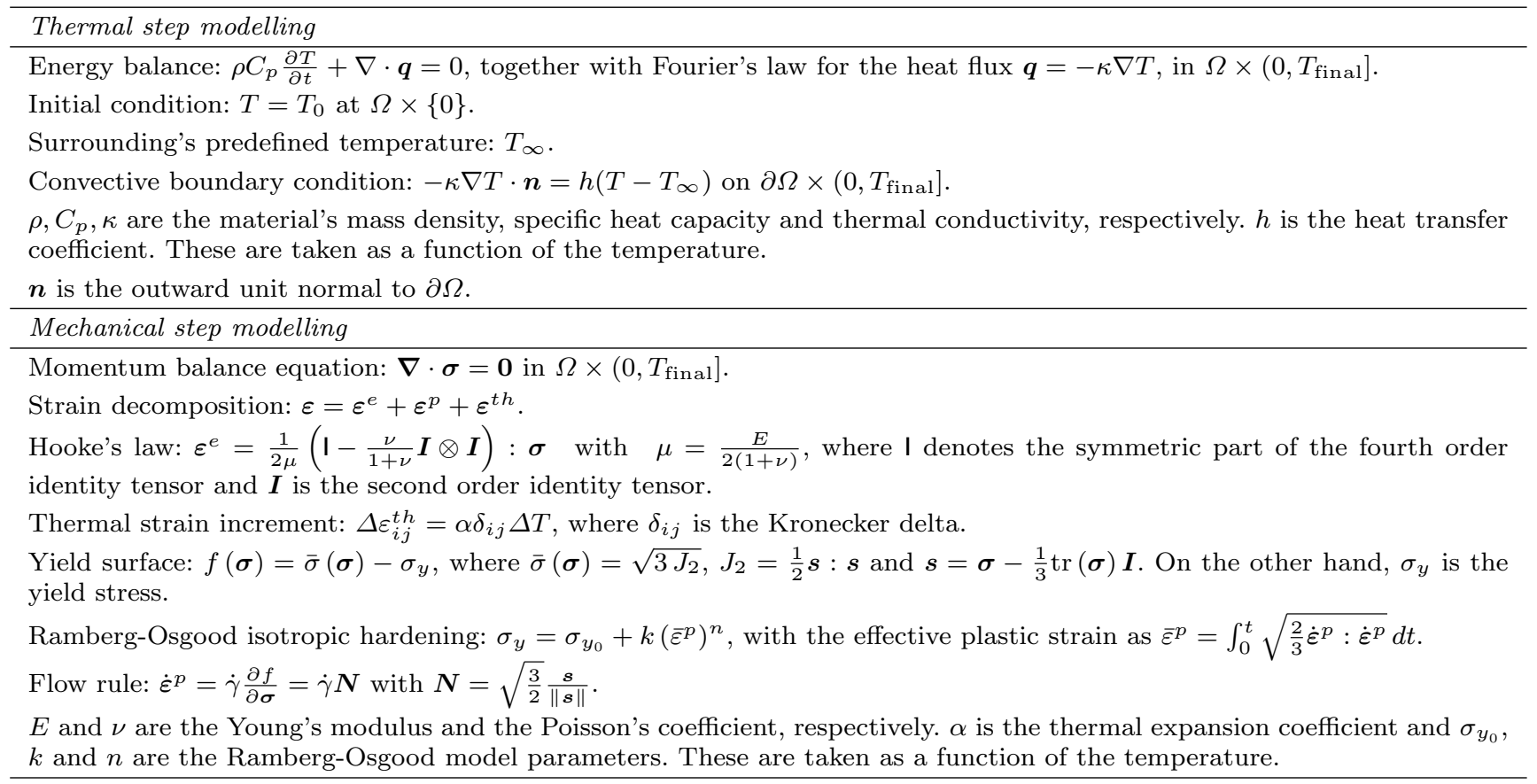

Table 2: Quenching model validation. Quenching process conditions from [?].

\begin{tabular}{llrr} 
Parameters & & & \\
\hline Initial temperature & $T_{0}$ & 467 & ${ }^{\circ} C$ \\
Quenching temperature (cold water) & $T_{\infty}$ & 20 & ${ }^{\circ} C$ \\
Quenching time & $T_{\text {final }}$ & 500 & $s$
\end{tabular}

and C3D8R for the mechanical step. Figure ?? depicts the geometry, boundary conditions and mesh of the numerical model.

\subsubsection{Validation of the residual stress prediction}

With the aim to validate the model, the longitudinal residual stresses $\sigma_{11}$ at the center of the plate $(x=L / 2$, $y=L T / 2)$ are compared against experimental measures. As explained in Section ??, the temperature evolution as a function of time is the first outcome before obtaining the residual stresses during quenching. The strong thermal gradient between the core and the surface of the part is depicted in Figure ??a for the initial 30 seconds of simulation. This is the source for the non-homogeneous plastic strains which produce residual stresses. Once thermal equilibrium is obtained, Figure ??b shows $\sigma_{11}$ along the thickness ( $z$ axis). We see that numerical predictions are in good agreement with experimental results, particularly in the internal area, whereas some border effect is observed near the surface. As a limitation, it should be noted that the model is not able to capture the local minima observed in the center of the plate, which is caused by an inhomogeneity of mechanical properties through-thickness [?,?] and produces in the 7XXX rolled plates the so called W-shape pattern of longitudinal yield strength through the ST direction [?].

For further assessment, Figure ?? shows a view on the $3 \mathrm{D}$ residual stress field. The RS pattern depends on the part's geometry and the thermal gradients generated during quenching [?]. As the model works under an isotropic material hypothesis, the stress components $\sigma_{11}$ and $\sigma_{22}$ are equal as they have the same dimension. In addition, they can be assumed as constant if the edge effects are neglected, which goes in line with the plane strain hypothesis used in some 2D models found in the literature [?,?]. However, with a 2D model we would not be able to study forged geometries and their complex stress states.

\subsection{Bending straightening simulation}

In this section, we consider a part with an internal residual stress field, coming either from the literature (in simple hot rolled parts mainly), from the quenching simulation model presented in section ??, or from any 

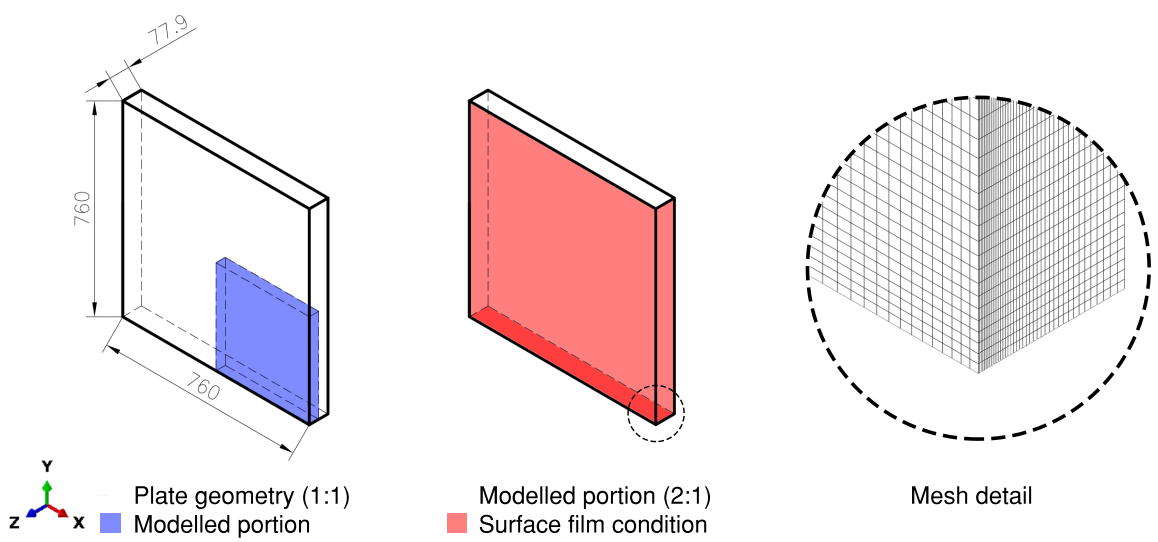

Fig. 4: Left: plate geometry with indication of the modelled portion. Center: in red, boundaries to which a mixedtype boundary condition is applied (adiabatic conditions applied on the symmetry planes). Right: detail of the mesh.

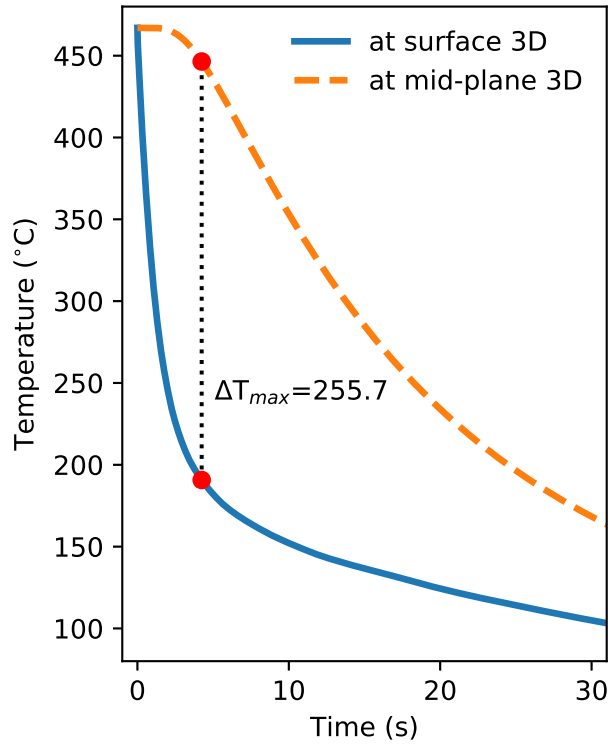

(a)

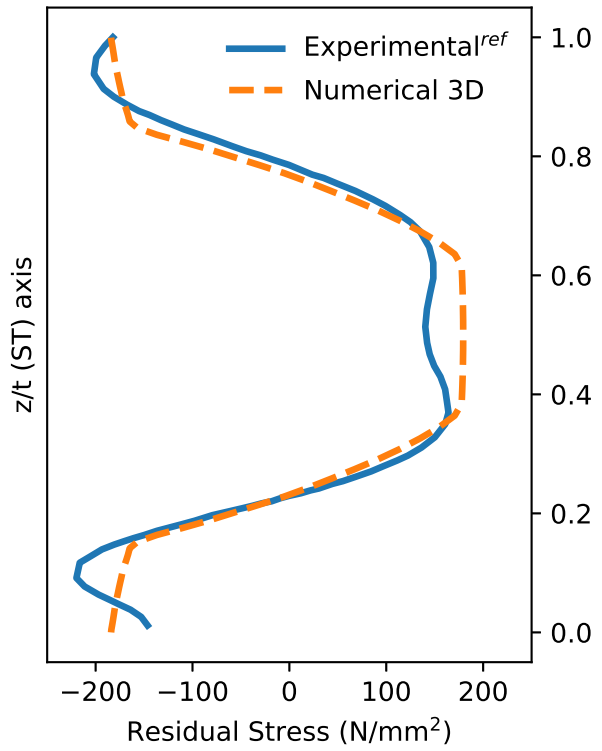

(b)

Fig. 5: Quenching simulation results at the center of the plate $(x=L / 2, y=L T / 2)$ : (a) Temperature evolution as a function of time; (b) Longitudinal residual stresses $\sigma_{11}$ along the thickness (short transverse direction) and validated against results from [?].

subsequent operation, such as stress relief or machining. In general, it is convenient to assume that at the outset of the simulation the part is stressed by some initial residual stresses, $\boldsymbol{\sigma}_{0}$. Considering that mechanical state, the objective is to develop a numerical model for bending straightening simulation.

\subsubsection{Elasto-plasticity modelling}

Bending straightening is an iterative procedure where the loading-unloading pattern can be considered as a cyclic behaviour. Therefore, it is crucial to take kinematic hardening into account for the accuracy of the results. To this end, we use the Chaboche model [?,?], which is synthetically presented in Table ?? together 


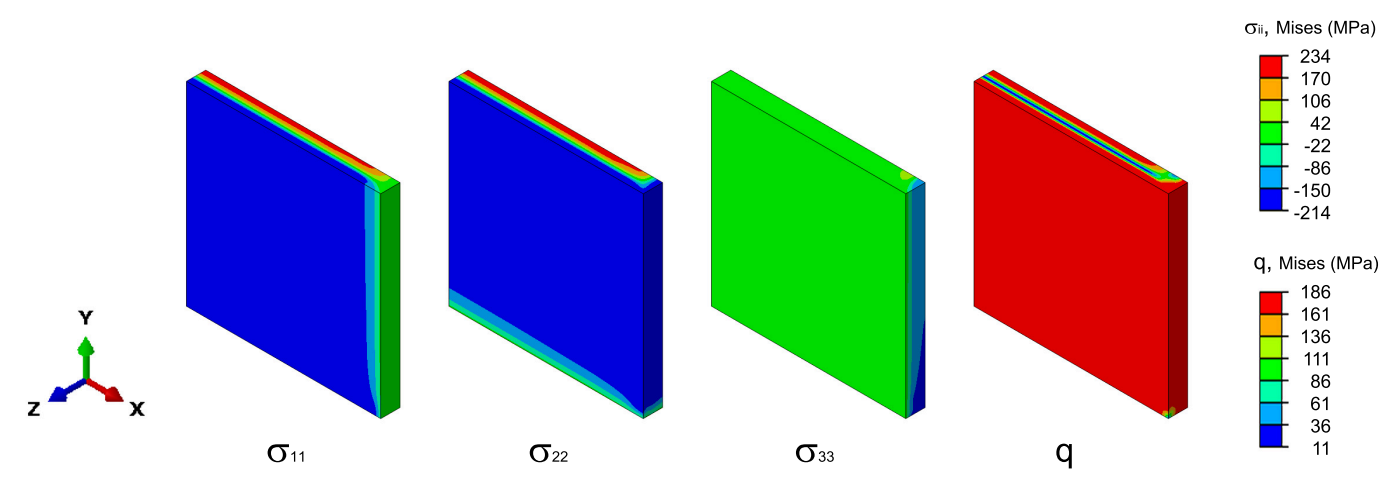

Fig. 6: Simulation results: 3D residual stress field in a rolled plate after quenching.

with the momentum balance equation, which has to be satisfied at each loading step.

Remark 1 (On the material modelling) A temperature -dependent version of the Chaboche model could have been used in section ?? for residual stress prediction after quenching. However, this kind of model is far more complex to characterize than the Ramberg-Osgood model, which in addition, proved to be accurate enough to capture the main mechanisms in residual stress creation.

Another important aspect that needs to be included in the numerical model is the interaction between the actuator that applies the load and the part, as well as the interaction between the part and the supports. The exact geometry of both the actuator and the supports depends on the experimental setup, and therefore a more precise description will be given in section ??. These are included in the model as rigid (undeformable) bodies. A Coulomb's frictional contact is considered between the actuator or the supports and the part. A penalty formulation is used for the numerical solution of the model. [?].

\subsubsection{Application to a four-point bending setup}

In order to validate the model presented in section ??, a four-point bending experiment was carried out. The geometry of the tested sample (machined from a hot rolled plate) as well as the experimental setup are shown in Figure ??.

The beam was plastically deformed by applying three cycles of loading and unloading. The amplitude of the cycles is $\pm 9.5 \mathrm{~mm}$, imposed by the hydraulic press, as a controlled displacement. Strain is recorded by two gauges placed at the top and bottom faces, labelled as J1 and J2, respectively. The sensors are located at the intersection of both longitudinal and transverse symmetry planes of the beam. In addition, displacements are recorded at two locations: at the mid-span of the beam and at the location where the force is applied. The force data acquisition is done by the hydraulic press head. In order to ensure repeatability, the experiment is performed in two samples, labelled as L1 and L2.

Concerning the numerical model, the plate was meshed with 56000 eight-node linear hexahedron elements (type C3D8R) and 64539 nodes. A Coulomb's friction coefficient $\mu=0.05$ was considered between the tool and the sample [?]. The material is an aluminium alloy AA7010, previously characterized by Airbus Central Research and Technology [?]. Material parameters are summarized in Table ??. The backstress tensor $\boldsymbol{X}$ in the Chaboche's model is described with three terms, i.e. $m=3$, see Table ??.

Finally, as discussed above, it is essential to have good knowledge on the residual stress field. As the tested sample is a rolled plate in a temper designation Txx51, meaning stress relief by stretching, it is expected to have a well-known residual stress profile, in the range of $\pm 30 \mathrm{MPa}[?, ?]$. The residual stress profile reported in [?] was considered in the simulation.

\subsubsection{Validation of the bending straightening model}

As a means to validate the simulation model, we use force versus strain measures generated during reshaping. Figure ??a presents a comparison of the experimental measures at gauge J1, for both specimens L1 and L2, against the numerical prediction. Figure ??b presents essentially the same results but at gauge J2. We first observe a very good repeatability between specimens L1 and L2, whose results match almost perfectly. Most importantly, a good agreement between the numerical results and experimental measures is observed in all cases. 
Table 3: Elasto-plastic model with kinematic hardening for bending straightening simulation

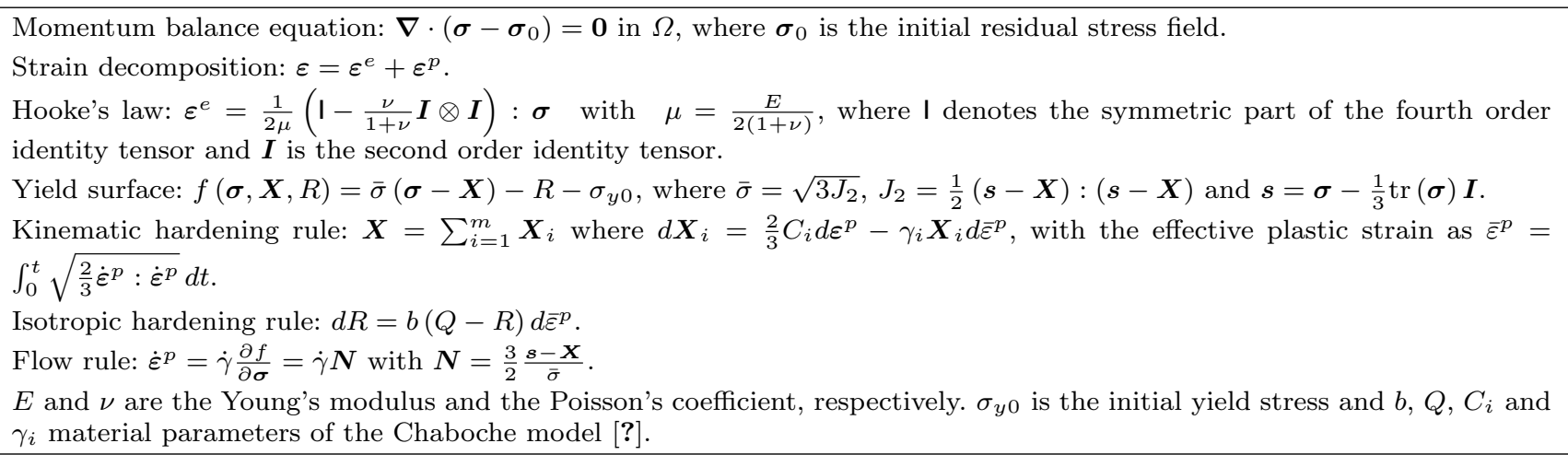

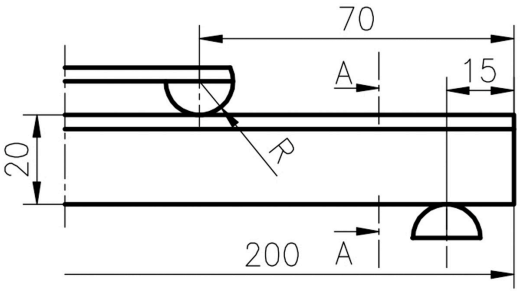

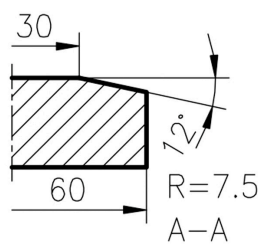

(a) Geometry of the sample

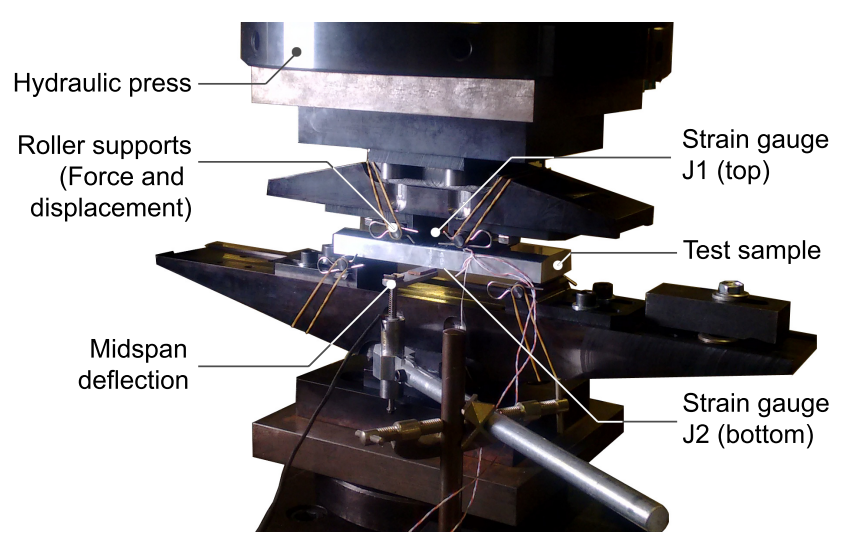

(b) Experimental setup

Fig. 7: Four-point bending experiment for the validation of the bending straightening model

Table 4: Chaboche's material model parameters for AA7010 [?]. All units are in MPa except for $\nu, b$ and $\gamma_{i}$ which are dimensionless.

\begin{tabular}{cccccc}
\hline$E$ & $\sigma_{y}$ & $\nu$ & $Q$ & $b$ & $C_{1}$ \\
\hline 73000 & 390 & 0.3 & -145.7 & 239.4 & 38973.6 \\
\hline$C_{2}$ & $C_{3}$ & $\gamma_{1}$ & $\gamma_{2}$ & $\gamma_{3}$ & \\
\hline 195910 & 3298.1 & 275.5 & 3134.6 & 23.4 & \\
\hline
\end{tabular}

\section{Numerical study of forged parts}

In this section, the numerical model presented and validated in section ?? is applied to the study of forged parts. As a reference geometry, we consider a beam with T-shaped cross-section, a simplified version of the cruciform, but still representative in terms of the thickness or the radius of the fillets.

Figure ?? shows the cross section of both the Tshaped beam and the bulk from which it is machined, with their geometric dimensioning. The final geometry is obtained through milling by removing $73 \%$ of the bulk material. As this step is done numerically, no information is provided regarding the finishing condition and cutting parameters.
An important parameter for the machining process is the so-called machining offset $\left(M_{o}\right)$, depicted also in Figure ??, which controls the positioning of the beam in the bulk. We shall analyse the strong influence of the machining offset on the resulting post-machining residual stress field, and by consequence, on the distortion. Figure ?? shows a parametrized four-point bending configuration, where several configurations are considered in terms of the distance between the supports and the distance between the loading points.

The simulation parameters are the same used in section ??, except for the geometry. 


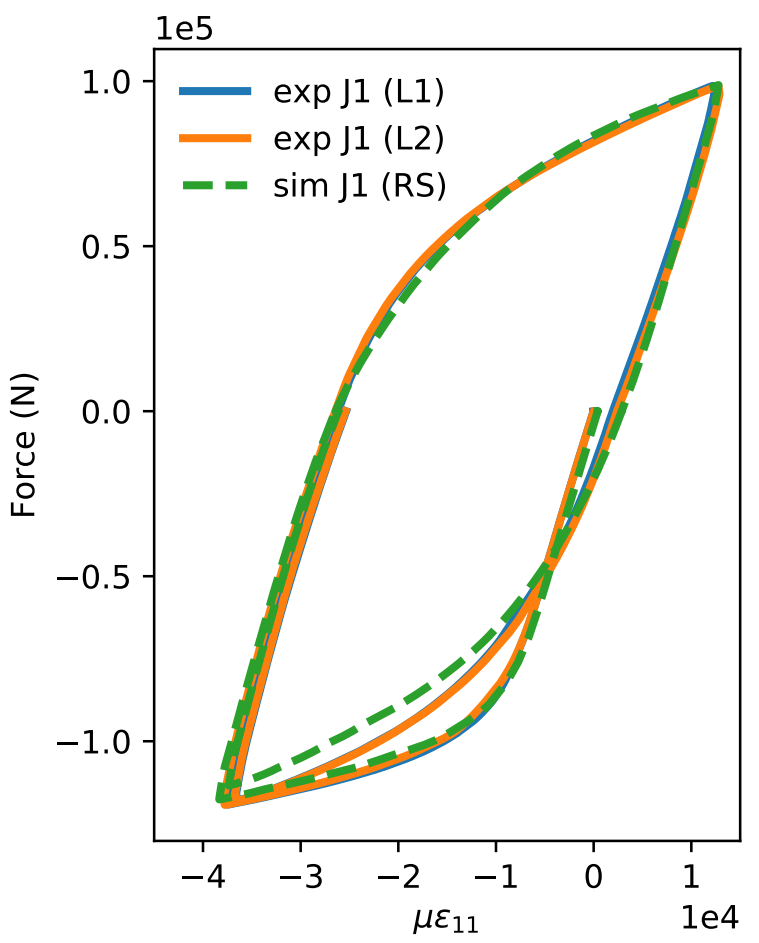

(a)

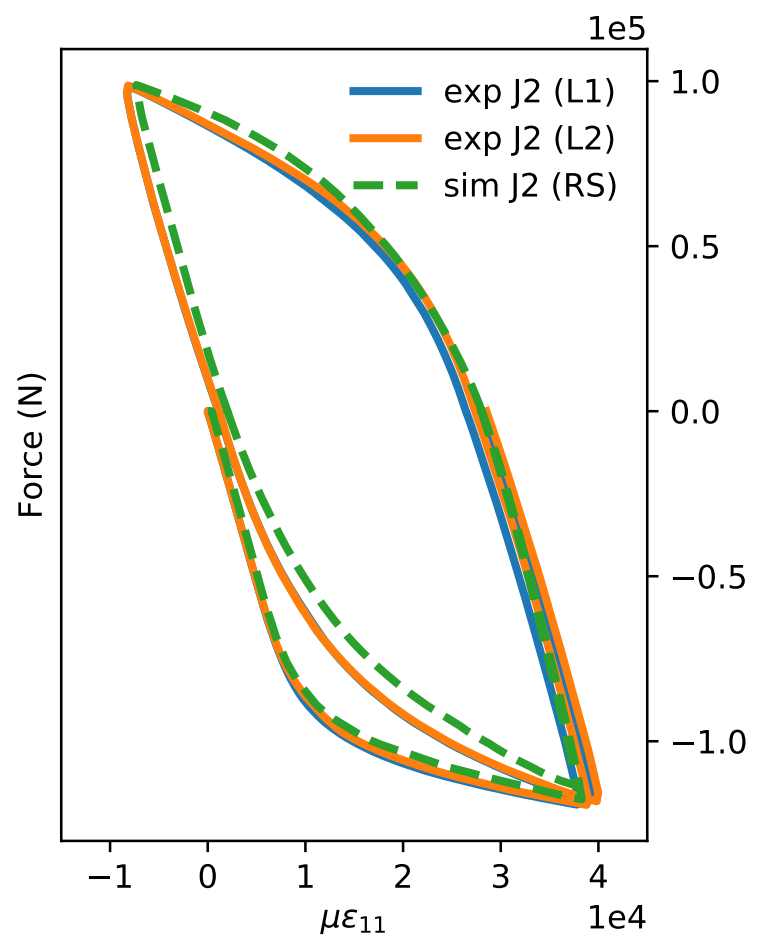

(b)

Fig. 8: Validation of the bending straightening simulation model via strain versus force measures: comparison of the simulation results (dashed line) and the experimental measures (solid line) for two specimens labelled L1 and L2. (a) Comparison for strain gauge J1 (located at top) and (b) Comparison for strain gauge J2 (located at bottom).

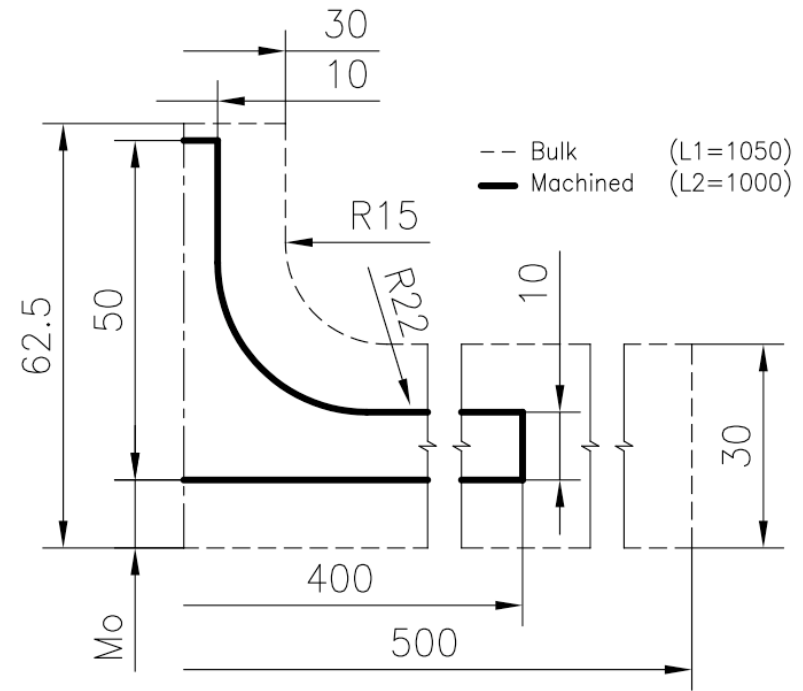

(a) Geometry definition of both the bulk and the machined part, as a function of the machining offset $M_{O}$

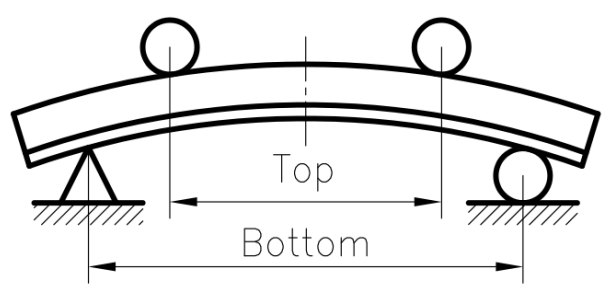

\begin{tabular}{|c|c|c|c|c|c|c|}
\hline Mo (mm) & \multicolumn{3}{|c|}{10} & \multicolumn{3}{c|}{5} \\
\hline Position ID & P1 & P2 & P3 & P4 & P5 & P6 \\
\hline Top (mm) & 300 & 600 & 300 & 900 & 900 & 600 \\
\hline Bottom (mm) & 900 & 900 & 600 & 300 & 600 & 300 \\
\hline
\end{tabular}

(b) Bending straightening setup and parametrization of the positioning of both top and bottom rollers

Fig. 9: Reference geometry for the simulation of bending straightening of forged parts: a beam with T-shaped cross section 


\subsection{Residual stress after quenching}

The thermo-plasticity model presented in section ?? is now applied to the T-shaped beam (bulk, i.e. before machining). Whereas the plate geometry in section ?? exhibited a nearly unidirectional (through thickness) heat conduction, the temperature field in the T-shaped beam is somewhat more complex to explain. To describe it in a simple manner, we consider control points in three main areas of the beam: the wing, the rib and the fillet. To each of these points, located on the surface, we associate an interior pairing point, with the objective of quantifying the temperature difference between the surface and the core. Figure ?? shows the positioning of the control points and compares the temperature evolution. Interior points are located following the normal to the outer boundary.

Temperature profiles are significantly different from one region to another, see Figure ??. The maximum temperature difference between internal and external control points takes place in the fillet. The curved zone is the last one to reach the thermal equilibrium, maximizing the development of non-homogeneous strains. Therefore, we can expect to have the maximum residual stresses in that region.

Figure ??a shows the residual stress field for the quenched bulk when the room temperature has been reached. As expected, the surface is subjected to compression stresses, which are equilibrated by tension stresses in the core. As the structural part is under a three-axial stress state, to know where plasticity has occurred, we use the von Mises yield criterion. As the yield stress $\sigma_{y}$ in the as-quenched state is $162 \mathrm{MPa}$, it can be appreciated on the bottom section of Figure ??a, how all the elements coloured in red have plastified and how its distribution is not homogeneous across the geometry.

\subsection{Residual stresses after machining}

After quenching, machining takes place to extract the T-shaped beam from the bulk. Material removal breaks the stress equilibrium producing a redistribution of residual stresses and, consequently, shape distortions. One of the main parameters of the machining step is the machining offset $\left(M_{o}\right)$, defined in the introduction of section ??, which controls the positioning of the T-shaped beam inside the bulk. We should therefore expect different post-machining distortion levels depending on the choice of $M_{o} \in[1,12] \mathrm{mm}$.

In order to provide an overall look on all possible shapes prior to reshaping, we carry out a parametric study on the effect of the machining offset.
Machining is modeled using the death-birth method [?], in which the material removal is simulated by deactivating the elements outside the machined geometry. The method requires to define the machined geometry as a subset in the mesh of the bulk.

Residual stresses introduced by machining have an impact in a superficial layer with a depth range of 250$300 \mu \mathrm{m}[?, ?]$, therefore, they must be included in the distortion simulation for parts with thin wall thickness $t<4 \mathrm{~mm}$ [?]. For this class of parts, the no inclusion of machining-RS can lead to a different pattern of distortion respect to the numerical prediction [?]. As we focus on forgings where the machined components are considered as thick walled, machining RS are not included, following the procedure described in [?]. As a consequence, the effects of clamping, cutting force and heat are not considered in the model.

As machining takes places after ageing, $\sigma_{y}$ evolves from $162 \mathrm{MPa}$ to $390 \mathrm{MPa}$ (see remark ??). Therefore, the redistribution of bulk RS, which are the main cause of distortion [?,?], are inside of the elastic region and it is possible to perform the machining operation in one single (deactivation) step. However, before arriving to that conclusion, the authors have considered different machining configurations, including a sequential deactivation by zones previous to obtain the final geometry and the results in terms of distortions were the same.

Remark 2 (On stress relief and ageing) Other manufacturing steps may take place after quenching and prior to the machining step, including stress relief and ageing. For simple geometries such as rolled plates and sheets, stress relief by stretching is usually carried out, whereas cold compression is preferred for forged parts $[?, ?]$. However, stress relief is often not possible for complex geometries [?]. Stress relief is not included in this study, as the main idea is to maximize the initial distortion caused by residual stress to place ourselves in the most adverse scenario. After the stress relief operation, the yield stress $\sigma_{y}$ is increased via a second heat treatment called precipitation hardening or ageing. This change of material properties is caused by a blockage of dislocations or defects in the crystal lattice [?]. It is reported that ageing does not affect the residual stresses [?]. Ageing is taken into account via material modelling with an increased yield stress $\left(\sigma_{y}=390 \mathrm{MPa}\right)$ (Chaboche kinematic hardening).

By varying the machining offset, two types of distortion are observed: bending along the longitudinal direction, also referred to as distortion type 1 , and wings closure, also referred to as distortion type 2 .

Figure ?? shows distortion after machining, both type 1 and type 2 , as a function of the machining offset. 

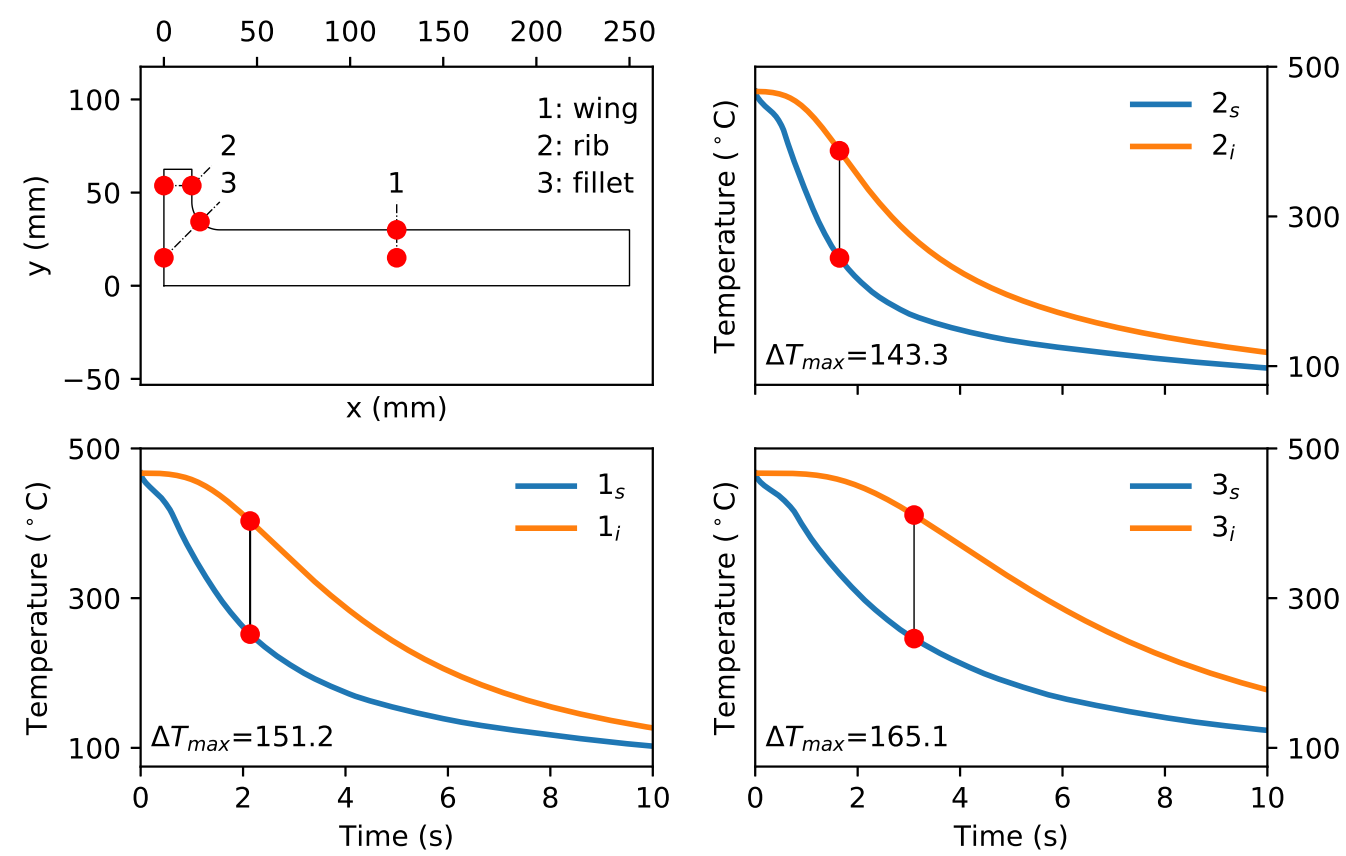

Fig. 10: Temperature evolution in the T-shaped beam (bulk) during the first 10 seconds of simulation at the (1) wing, (2) rib and (3) fillet. The cross section corresponds to the symmetry plane $z=0$ and the subscripts $s$ and $i$ refer to the surface and internal points.

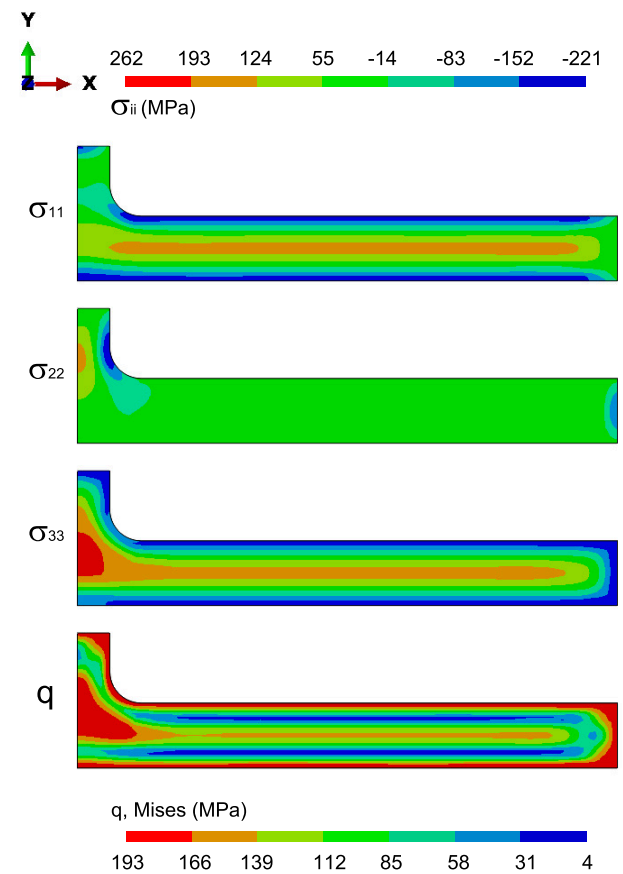

(a)
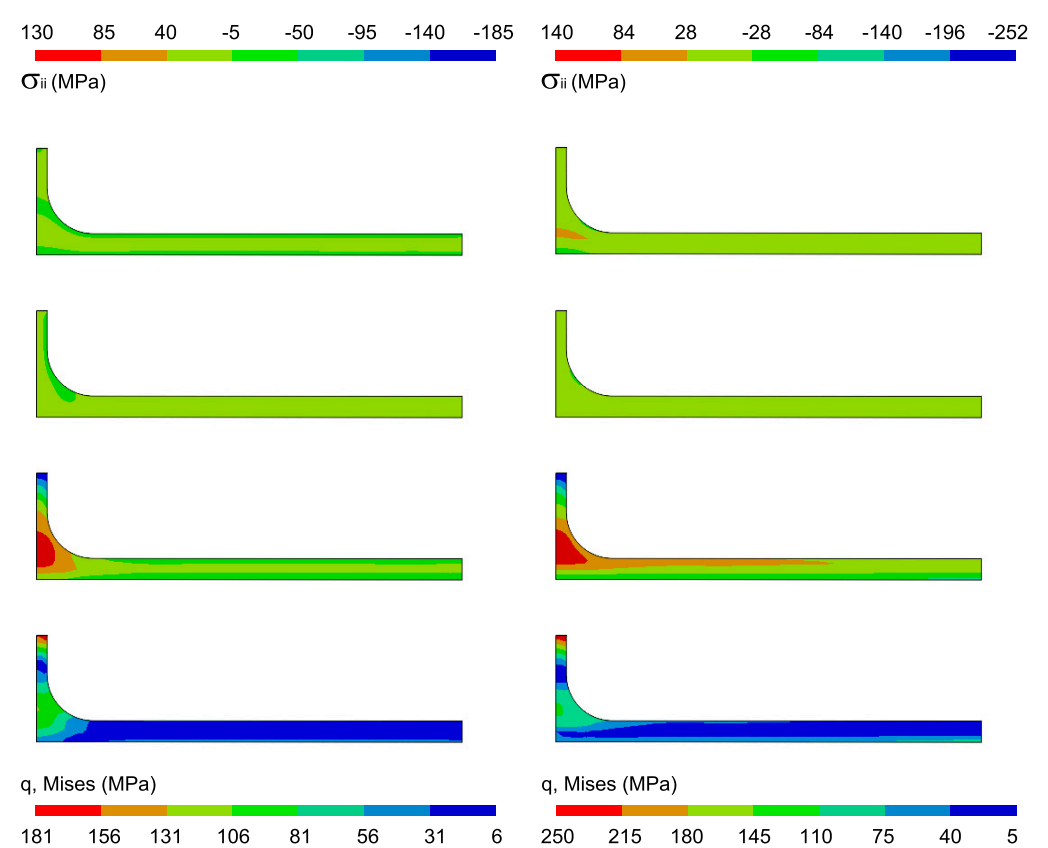

(b)

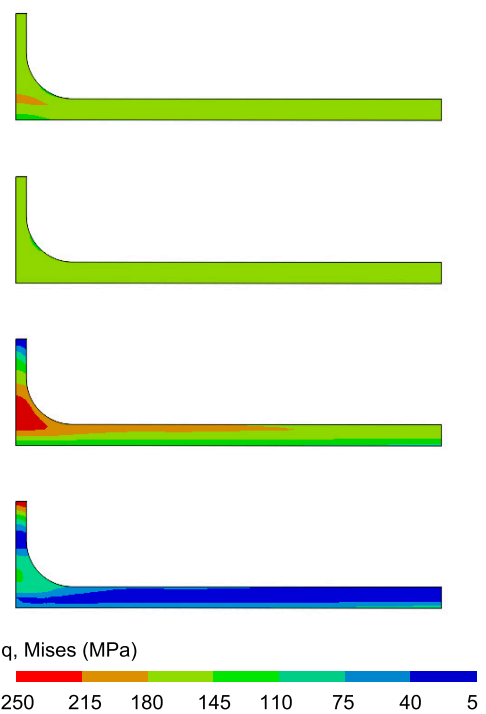

(c)

Fig. 11: Residual stress distribution in the T-shaped beam: (a) after quenching (bulk), (b) after machining with $M_{o}=10 \mathrm{~mm}$ (case A) and (c) after machining with $M_{O}=5 \mathrm{~mm}$ (case B). All the snapshots are taken in the symmetry plane $z=0$ and presented in the undeformed configuration. 
It can be seen that for lower offset values bending distortion takes predominance over wings closure, whereas the opposite is observed for higher values of the machining offset. In general, both distortions tend to become negative as machining offset increases, meaning that bending inverts and wings open instead of closing. To illustrate the behaviour, we select two specific values of the machining offset parameter, labelled as configuration $\mathrm{A}$ and $\mathrm{B}$, which correspond to $M_{o}=10 \mathrm{~mm}$ and $M_{o}=5 \mathrm{~mm}$, respectively. Figures ??b and ??c show the stress components for the machining cases $\mathrm{A}$ and $B$, respectively. Note that significantly different stress distributions are obtained by modifying $M_{o}$ only.

\subsection{Reshaping diagrams for bending straightening}

We shall now concentrate on distortion of type 1 , the only one that bending straightening can deal with. We measure distortion relative to the longitudinal axis of the part. Let $\mathbf{z}$ and $\mathbf{z}_{\text {nom }}$ be the coordinates of longitudinal axis of the distorted and the nominal (undistorted) part, respectively. It is implicit that $\mathbf{z}$ is measured in the deformed state. Let $\mathbf{n}$ be the unit normal in the bending plane to the longitudinal axis at $\mathbf{z}_{\text {nom. }}$. Distortion of type 1 is using a peak-to-peak amplitude measure:

$\delta=\max (d)-\min (d) \quad$ with $\quad d=\left(\mathbf{z}-\mathbf{z}_{\text {nom }}\right) \cdot \mathbf{n}$.

In the case of the T-shaped beam, the longitudinal axis of the undistorted part can be made coincident with $\mathbf{z}_{\text {nom }} \equiv \mathbf{0}$. The bending plane is the plane $z=0$, and therefore the normal is $\mathbf{n}=(0, \pm 1,0)$, the sign being arbitrary.

Assume a fixed machining offset, leading to an initial distortion $\delta_{0}$, which is assumed known as it can be measured. For a given reshaping configuration (i.e. rollers positioning), the bending straightening operation consists in guessing the stroke $y_{s}$ (equivalently, bending load) that should be imposed to minimize distortion after unloading. Note the parametric dependence of distortion as a function of stroke, $y_{s} \mapsto \delta\left(y_{s}\right)$. To assist operators to make their decision, we may rely on the simulation model developed in section ??. By sweeping over the range of stroke values, and carrying out a loading-unloading cycle simulation for each stroke, we obtain the reshaping diagram shown in Figure ??.

Three distinct areas can be identified. For low values of stroke (zone A), the part remains inside of the elastic domain and consequently distortion remains at its initial value, $\delta \approx \delta_{0}$. A reduction of the initial distortion is only possible when plasticity takes place. Here, the new residual stresses generated during the reshaping operation counteract the previous stress state. We labelled this region as zone B in Figure ??, where $\delta<\delta_{0}$. Inside this zone, there is an optimum configuration, where distortion is minimized, (i.e. $y_{s}^{o p t} \mapsto \delta_{\text {opt }}$ ). Finally, zone $\mathrm{C}$ comes after reaching the minimum, it is characterized by increasing distortions for higher stroke.

Figure ?? shows the deformed shape (longitudinal axis only) for three different values of stroke, one for each of the identified region. Specifically, the optimum is shown for zone B. The location of the bottom roller is the reference line. Green and red areas correspond to the points above and below the reference line, respectively (also, these correspond to the sign of $d$ as defined in Eq.(??)).

Note that the reshaping diagram is a very convenient tool to assist the operator: once it is made available, the reshaping operation reduces to select the optimal bending load that minimizes the remaining distortion. Unfortunately, computing the reshaping diagram requires a precise knowledge on the residual stress field, which is not a trivial task. We shall discuss in section ?? an alternative approach based on the idea that, to minimize the distortion, we may not need a precise knowledge of the residual stress field, but only its influence on the reshaping diagram.

\subsection{Parametric study of the reshaping diagram}

The reshaping diagram in Figure ?? was computed for a fixed machining offset and rollers positioning. To provide further insight, in this section we consider six possible configurations by combining two values of the machining offset parameter and three rollers positioning. Each configuration is labelled as $\mathrm{P} 1$ to $\mathrm{P} 6$. The specific values for each of these is given in Figure ??. Note that rollers positioning is inverted in configurations $\mathrm{P} 4$ to P6, compared to P1 to P3, because the deformed shape is also inverted.

Figure ?? shows the reshaping diagrams obtained for each of the six configurations. The same three areas described above can be distinguished. Figure ?? a shows configurations P1 to P3, which correspond to a machining offset of $M_{o}=10 \mathrm{~mm}$. Figure ??b shows configurations $\mathrm{P} 4$ to $\mathrm{P} 6$, which correspond to a machining offset of $M_{o}=5 \mathrm{~mm}$.

One of the main conclusions that can be drawn from Figure ?? is that the position of the rollers has significant impact on the reshaping diagram. For instance, let us consider configurations P1 and P3. For both configurations, the internal rollers (loading) are positioned at $300 \mathrm{~mm}$, whereas the external rollers (supports) are at $900 \mathrm{~mm}$ and $600 \mathrm{~mm}$, respectively. Both configurations reach a similar value of optimal distortion, but the 


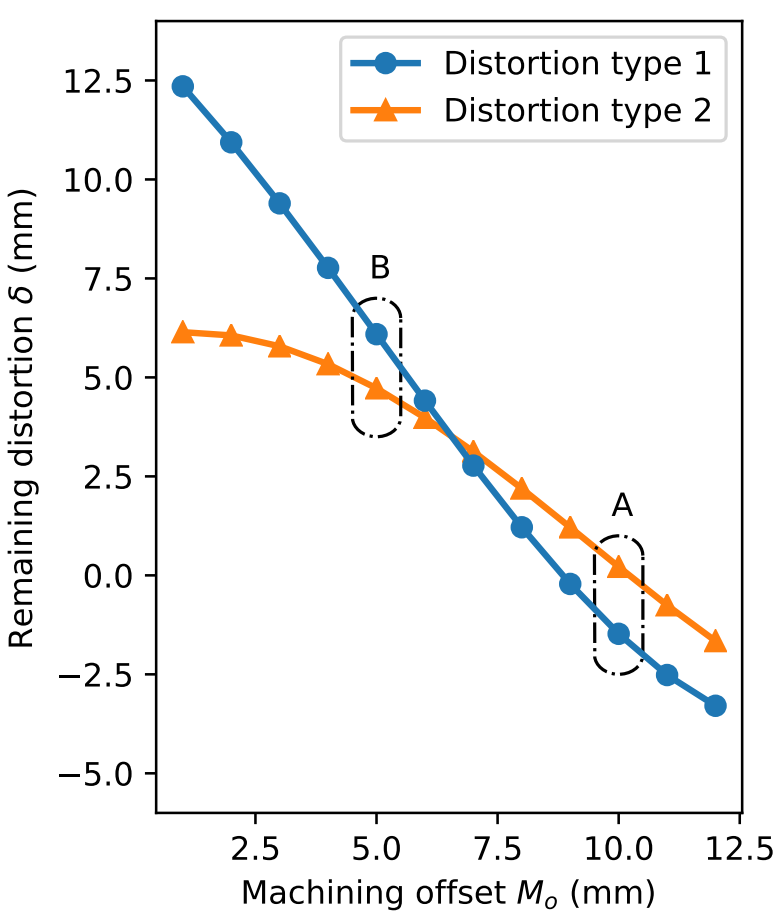

(a)

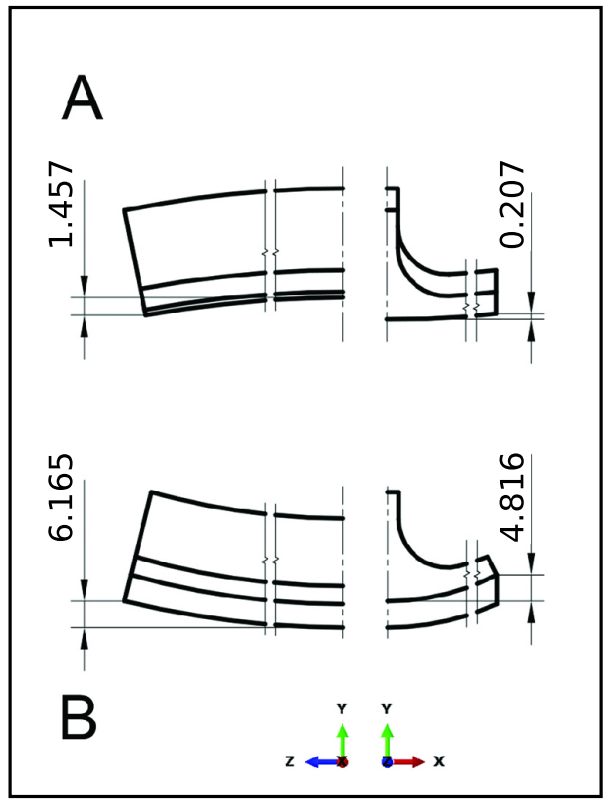

(b)

Fig. 12: T shaped beam model. (a) Distortion after machining as a function of machining offset $M_{o}$. (b) Distorted shape for case A $\left(M_{o}=10 \mathrm{~mm}\right)$ and case $\mathrm{B}\left(M_{o}=5 \mathrm{~mm}\right)$.

stroke to be applied is P1 almost doubles that of P3. This is to be expected, as configuration P1 is less rigid respect to $\mathrm{P} 3^{1}$. As a consequence, $\mathrm{P} 1$ requires more deflection to reach plasticity. Another important effect that can be noticed is related to the sensitivity of the optimum. By sensitivity we mean the change in distortion as the optimal stroke is affected by some perturbation, i.e. $y_{s}^{\mathrm{opt}}+\varepsilon \mapsto \delta_{\text {opt }}+\Delta \delta$. Clearly, the configuration $\mathrm{P} 1$ is less sensitive to perturbations in the stroke and therefore, in practice it should be easier to achieve. This effect becomes even clearer if the comparison is made between $\mathrm{P} 4$ and $\mathrm{P} 5$.

\footnotetext{
1 For a beam, the force-displacement diagram can be used to calculate the stiffness of the structure, which is defined by the Equivalent Young's Modulus $E_{0}$ and corresponds to the slope of the curve. In a four point bending configuration, $E_{0}$ is defined as $E_{0}=24 E I /\left[\left(3 L^{2}-4 a^{2}\right) a\right]$, where $E, I, L$ and $a$ correspond to the Young's Modulus, the inertia of the cross section, the span between external supports and the distance between one single external and internal roller, respectively. For example, if we express $L$ and $a$ in terms of the span of P1 $(900 \mathrm{~mm})$, both pair of parameters are $(L, L / 3)$ for P1 and $(2 L / 3, L / 6)$ for P3, respectively. By replacing both sets of values in the previous expression, we found that $E_{0(P 1)}=$ $11 / 46 E_{0(P 3)}$.
}

\section{Residual stress free diagrams for simulation-assisted reshaping}

As discussed in section ??, reshaping diagrams can be very helpful, provided that the residual stress field is known. However, at least two drawbacks can be outlined:

- Residual stress computation requires running sequential simulation models that account for all manufacturing steps prior to reshaping. Therefore, one needs to keep track of all process conditions and part history to run accurate predictions. Moreover, this process can be computationally intensive and time-consuming.

- Uncertainties linked to modelling and process conditions are likely to have a significant impact. To compute a trustworthy residual stress field, one would need to assess carefully how uncertainties propagate through the simulation chain.

To overcome these issues, we propose an alternative approach. The underlying idea is that to minimize the distortion we may not need a precise prediction of the residual stress field, but only its influence on the re- 


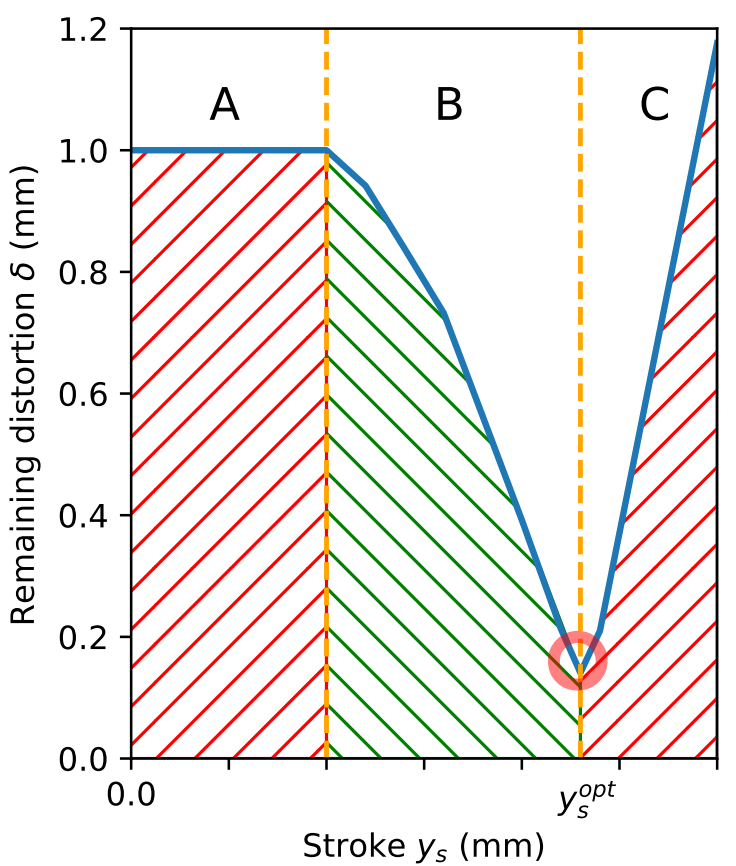

\section{Zones}

A. No reshaping is performed $\delta \approx \delta_{0}$

$B$. Reshaping takes place $\delta<\delta_{0}$

(a)

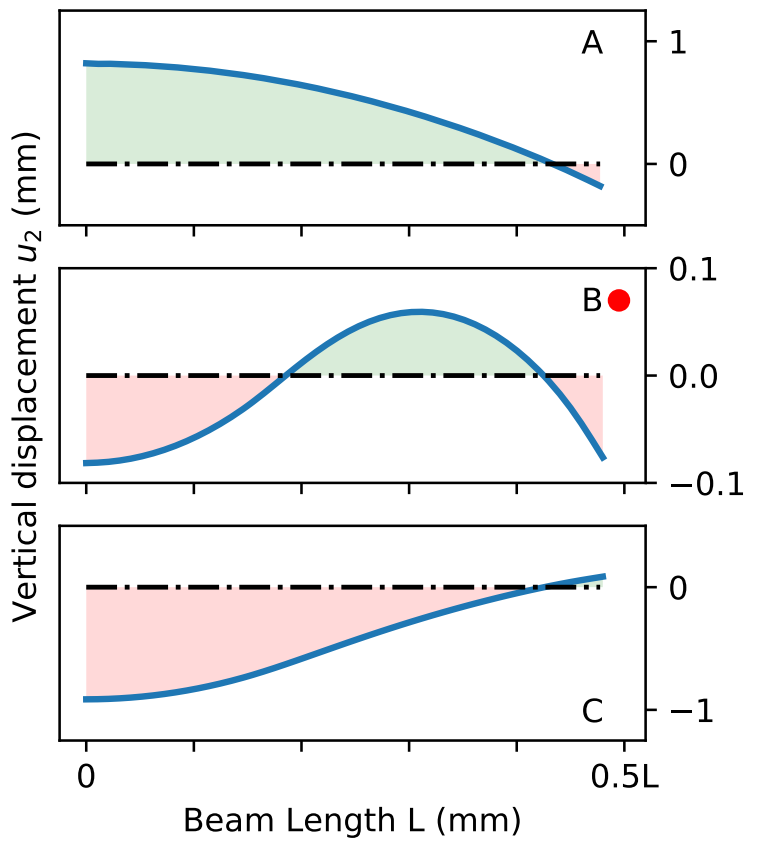

Optimum configuration $\delta=\delta_{\text {min }}$ C. Distortion increment $\delta>\delta_{0}$

(b)

Fig. 13: Reshaping diagram (scheme). (a) The three characteristic regions of the reshaping diagram: elastic area of no repair (A), repairing area (B) and inversion area (C). (b) Shape after reshaping for values of the stroke in each reshaping region.

shaping diagram. Let us consider the parametric study already presented in section ??. For each of the configurations $\mathrm{P} 1$ to $\mathrm{P} 6$, we compute the reshaping diagram of a distorted part but neglecting the residual stress field. That is, we keep the distorted geometry after machining but suppress the residual stress field. The results are shown in Figure ??.

From Figure ?? we can conclude that neglecting the residual stresses results only in a shift of the reshaping diagram: $\delta\left(y_{s}\right) \approx \delta_{\mathrm{RSF}}\left(y_{s}^{\mathrm{RSF}}+\Delta y_{s}\right)$. In other words, the overall behaviour including a realistic $3 \mathrm{D}$ residual stress field can be retrieved from the residual stress free diagram, provided that we are able to devise a strategy to identify the appropriate offset $\Delta y_{s}$.

The reshaping offset can be negative, as in Figure ??a, or positive, as in Figure ??b. To explain this behaviour, let us study the P5 configuration. Here, as the initial distortion has a $\mathrm{U}$ shape, bending is applied up- wards and therefore, tension is induced along the rib. However, the rib is initially in compression due to the residual stresses (see $\sigma_{33}$ in Figure ??c). As consequence of this inversion of stresses, more stroke needs to be applied in order to reach the yield surface, compared to the residual stress free case. As a general rule, if the stresses generated during reshaping oppose the initial residual stresses, the offset will be positive; otherwise, it will be negative.

The residual stress free approach has many advantages:

- It uses as the main input the initial distortion after machining, which unlike stresses, can be measured on a systematic basis.

- The underlying numerical model is rather simple, as it only needs to account for the bending straightening step. The computational cost is drastically reduced. See Figure ?? for comparison. 


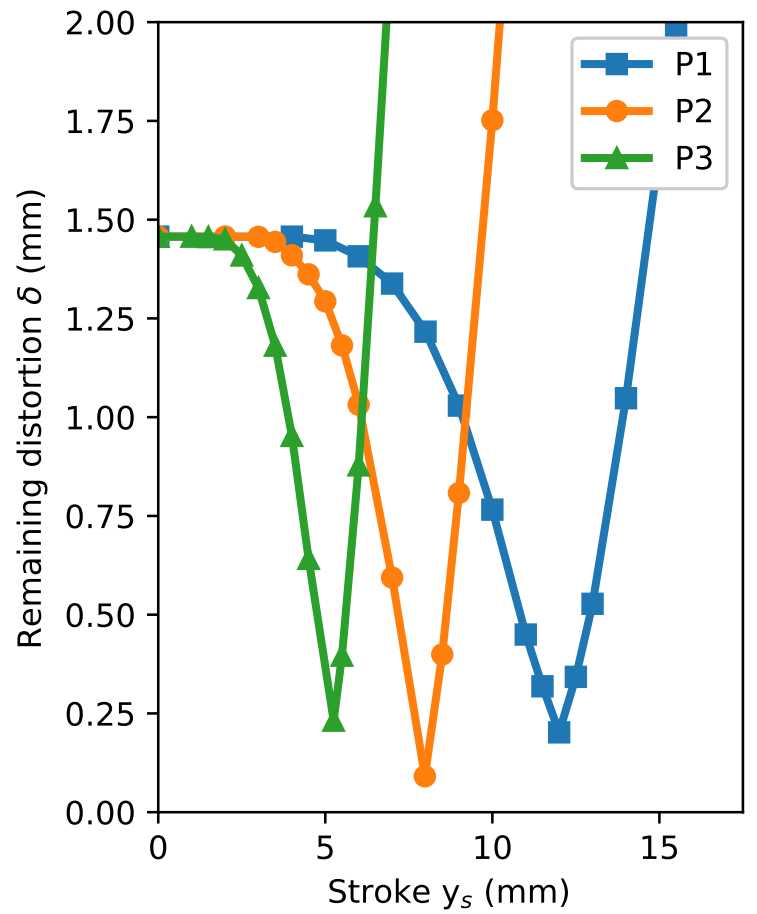

(a)

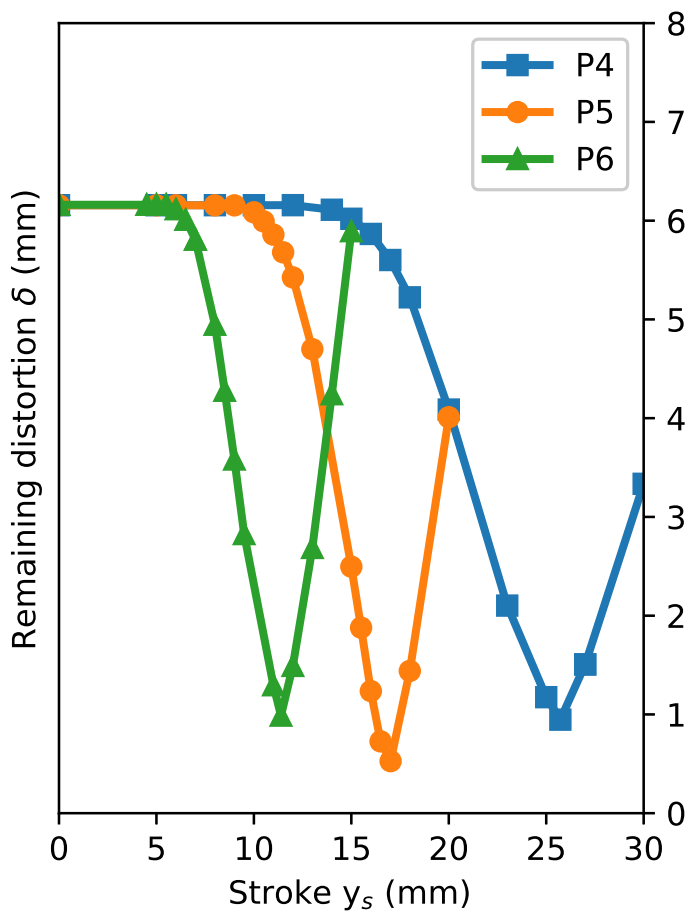

(b)

Fig. 14: Parametric study of the reshaping diagrams for six combinations of machining offset and the rollers positioning (values given in Figure ??): (a) $M_{o}=10 \mathrm{~mm}$ (case A) and three rollers positioning. (b) $M_{o}=5 \mathrm{~mm}$ (case B) and three rollers positioning.

- Modelling involves less parameters and, therefore, limited uncertainty. Furthermore, we can deal with uncertainties via the reshaping offset, to be identified experimentally.

4.1 Linear unloading path in the force-displacement diagram

In this section, we introduce the linear unloading path hypothesis, which is key for offset calibration. Our goal is to calibrate the offset without actually having to perform loading-unloading cycles, which would be very impractical. The linear unloading is a simplification hypothesis to anticipate springback without actually unloading the part. The idea is to approximate the unloading path in the force-displacement diagram (which is generally non-linear) with a linear behaviour ${ }^{2}$. The slope for such linear unloading (sometimes referred to as the equivalent Young's modulus) is computed in the linear part of the force-displacement diagram as $\Delta F / \Delta u$,

2 Not to be confused with the stress-strain diagram, where unloading is linear with slope equal to the Young's modulus. during loading and before yielding. This is a common engineering practice already well-documented in the literature $[?, ?, ?]$.

To assess the validity of the linear unloading simplification, let us consider the rolled plate studied in section ??. The linear unloading hypothesis is tested at two locations: at the mid-span of the plate and at the section where the loading is applied, with an imposed stroke is $y_{s}= \pm 9.5 \mathrm{~mm}$. Both force-displacement diagrams are represented in Figure ??.

A linear unloading path is represented at the final position of each cycle. Although it can be seen that the experimental unloading path follows a non-linear trajectory, for reshaping we are only interested in two points: where to stop the loading curve and the final distortion value in the part. Therefore, by using the linear approximation of the unloading path, both points can be tracked easily.

Further insight on the linear unloading hypothesis can be found in literature [?], where the authors state that when a complete unloading is performed after applying a bending moment $M$ to a beam, if unloading does not cause reverse yielding, then the unloading pro- 


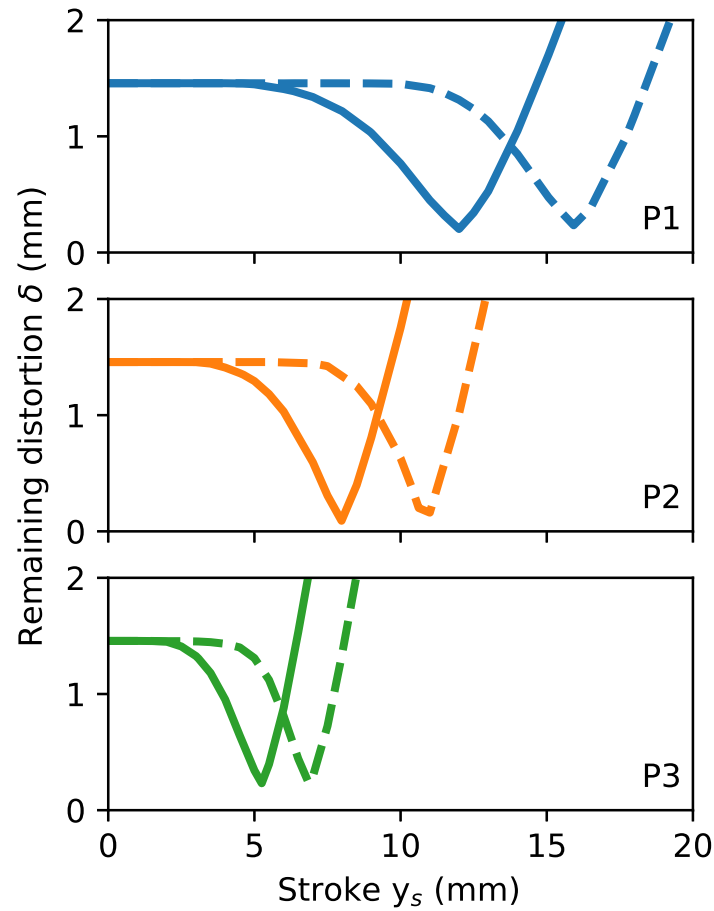

(a)

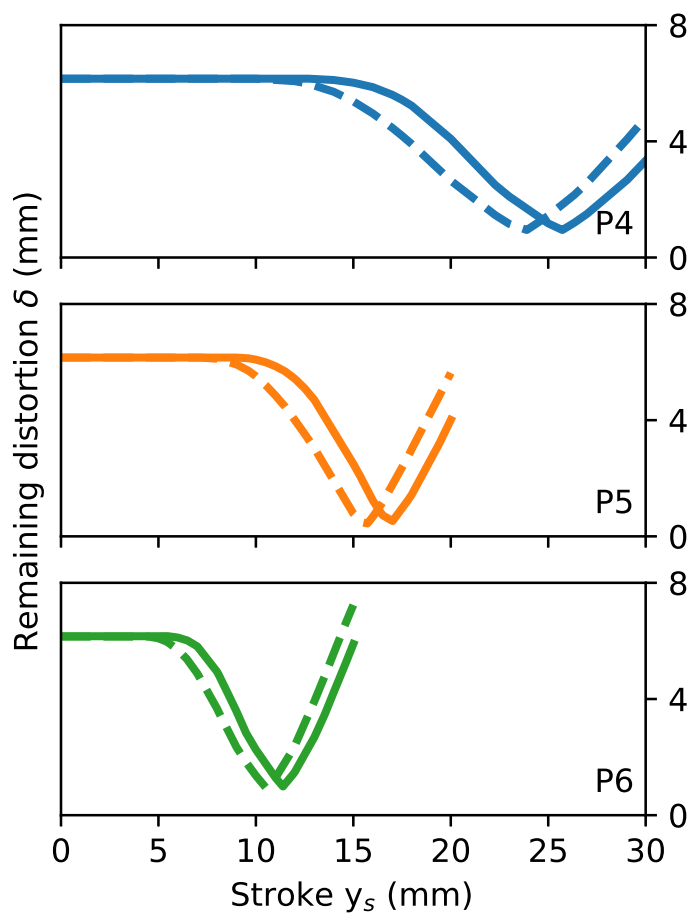

(b)

Fig. 15: Residual stress free reshaping diagrams (dashed line) against true reshaping diagrams that account for full 3D residual stress field (solid line), for six different reshaping configurations.

cess is equivalent to the elastic effect caused by applying $-M$ to the beam. Therefore, the key is to not produce reverse yielding. This phenomenon is present in metal sheet forming [?], very common in the automotive industry for instance. However, large structural elements in the aircraft industry can be considered as thick walled, and the levels of strain under reshaping are less likely to develop reverse yielding. Therefore, the unloading path can be approximated to a linear behaviour.

To further assess the validity of the linear unloading simplification, we consider now the T-shaped beam. The force-displacement diagram is generated for reshaping configurations $\mathrm{P} 1$ and $\mathrm{P} 5$. These are represented in Figures ?? and ??, respectively. Linear unloading is represented for different stroke values.

As it can be seen, the linear unloading path approximation is valid for both reshaping configurations. While P1 configuration follows naturally a linear path, P5 unloading is clearly non-linear. However, both the linear and the non-linear unloading path end nearly at same point. This is crucial, as the end point can be used to determine the distorted value after unloading.
In terms of relative error $E_{r e l}$, the linear unloading hypothesis causes an error of less than $10 \%$ for the final displacement respect to the FEM result, which proves to be a good approximation for industrial applications. The corresponding values for configuration P1 and P5 are summarized in Table ??. Finally, if both configurations are considered as the extreme values for the interval $M_{o} \in[5,10] \mathrm{mm}$ in the initial distortion diagram (see Figure ??a), it is expected that the linear unloading hypothesis remains valid for intermediate values as well.

\subsection{Reshaping offset calibration}

In this section, we propose a strategy, based on the linear unloading simplification, in order to calibrate the reshaping offset from experimental force-displacement measures.

Figure ?? summarizes the process for configurations P1 and P5. Since we do not have experimental measures, we shall assume the reshaping diagram (with residual stresses) as the ground truth for comparison. 


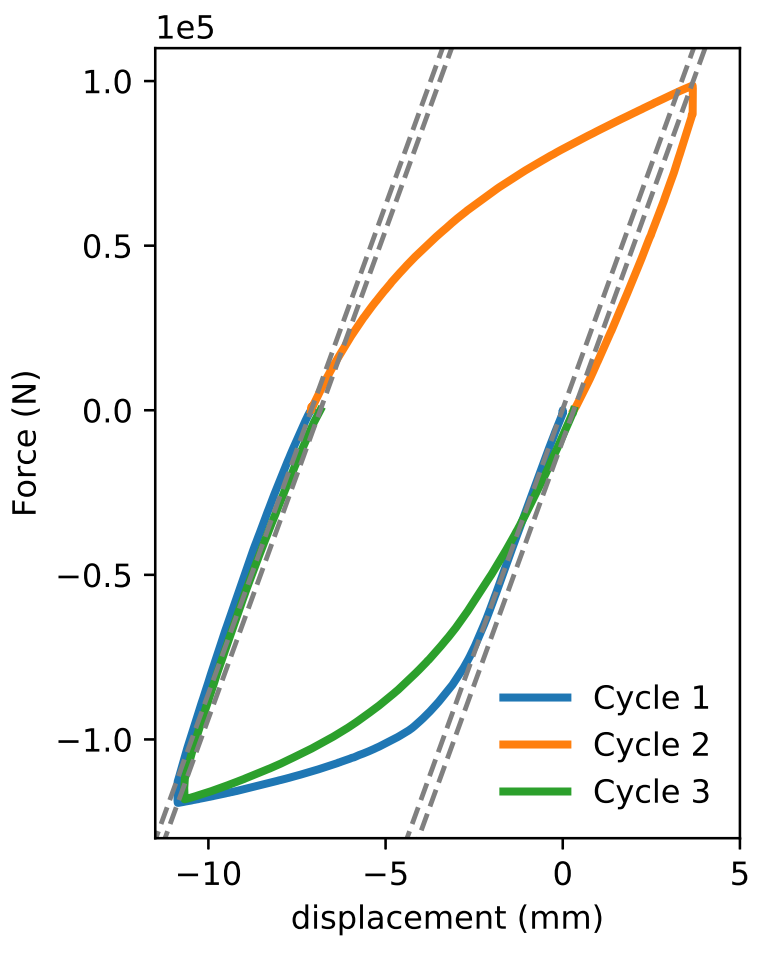

(a)

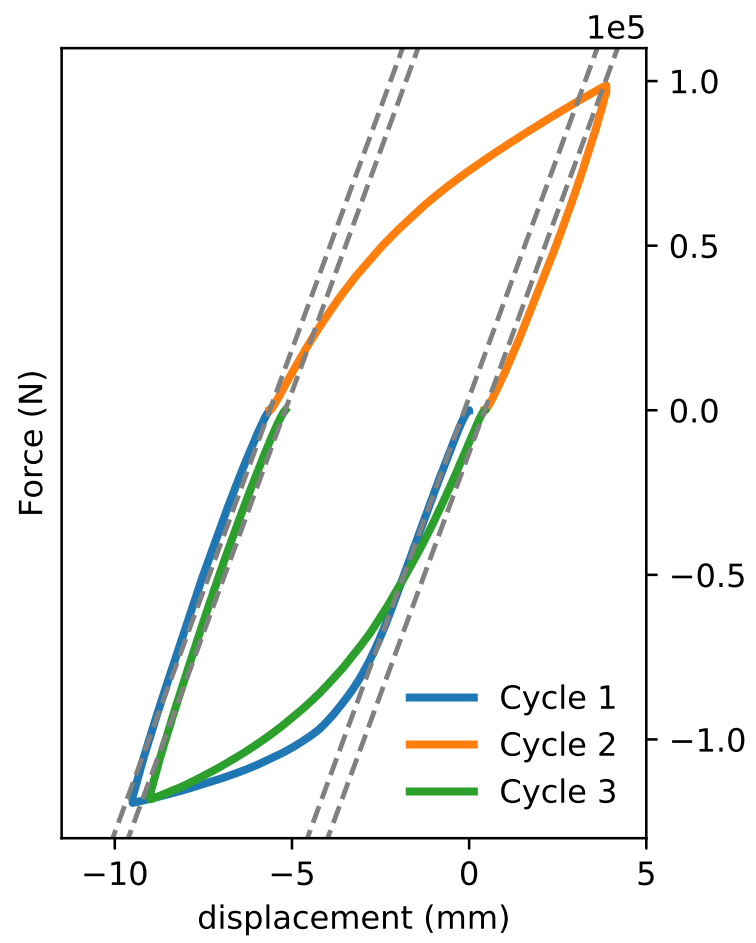

(b)

Fig. 16: Testing the linear unloading hypothesis. Force versus displacement diagram for the rectangular rolled plate (specimen L2). Experimental curves (solid lines) vs linear unloading path (dashed lines). (a) At midspan $L=100$ $\mathrm{mm}$ and (b) At the section where the load is applied $L=70 \mathrm{~mm}$ (see Figure ??).

Table 5: Final displacement for a given stroke $y_{s}$ during reshaping configurations P1 and P5: comparison between the non-linear (FEM) and linear unloading path (LU).

\begin{tabular}{lccccccc} 
Parameter / Configuration & \multicolumn{7}{c}{$\mathrm{P} 1$} \\
\hline Stroke $y_{s}$ & 12 & 14 & 16 & 15 & 17.015 & 20 & $\mathrm{~mm}$ \\
Displacement FEM & -1.280 & -2.182 & -3.268 & 3.171 & 5.376 & 8.758 & $\mathrm{~mm}$ \\
Displacement LU & -1.177 & -2.051 & -3.107 & 3.095 & 5.051 & 8.631 & $\mathrm{~mm}$ \\
Relative Error $E_{\text {rel }}$ & 8.1 & 6.0 & 4.9 & 2.4 & 6.0 & 1.5 & $\%$
\end{tabular}

First, we compute the reshaping diagram under a residual stress free state. This is defined as the off-line stage for our problem, where all the numerical simulations are performed. Now we start loading the part by increasing the stroke (controlled displacement). We also record the applied force by the press head. By applying the linear unloading simplification, we obtain an approximation of the distortion after unloading. This is depicted in green in Figure ??.

The actual reshaping diagram is a priori unknown, in principle, it can only be obtained by performing loading (apply stroke) then unloading (measure distortion) cycles. This trial and error procedure describes the industrial practice today and it is undesired.
Instead, we propose to use force-displacement measures, which are rather standard in bending operations, in combination with a linear unloading path hypothesis, to derive an approximation of the experimental reshaping diagram. This second step is referred as the on-line stage.

Once we enter the zone B of the reshaping diagram, we can start noticing the difference in response between the residual stress free diagram and the experimental approximation. As soon as the difference becomes steady, the offset can be determined. From the knowledge of the offset, the optimal stroke can be approximated as: $y_{s}^{\mathrm{opt}}=y_{s}^{\mathrm{opt}, \mathrm{RSF}}+\Delta y_{s}$.

Following with configurations $\mathrm{P} 1$ and $\mathrm{P} 5, \Delta y_{s}$ is presented in Table ??.As a result, the applied stroke $y_{s}^{o p t}$ 


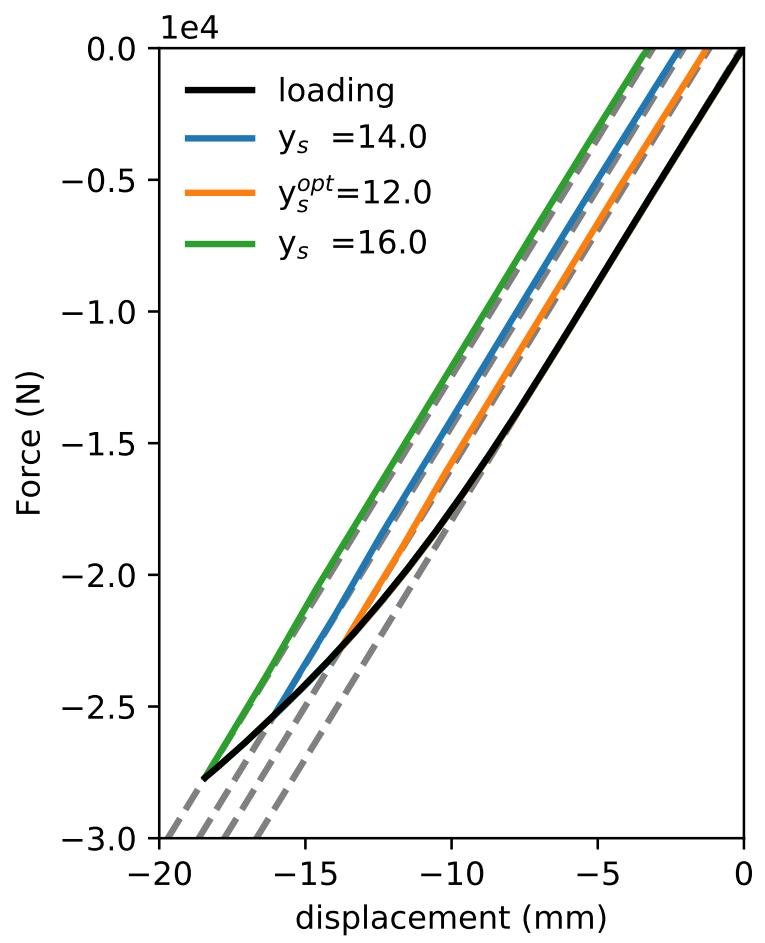

(a)

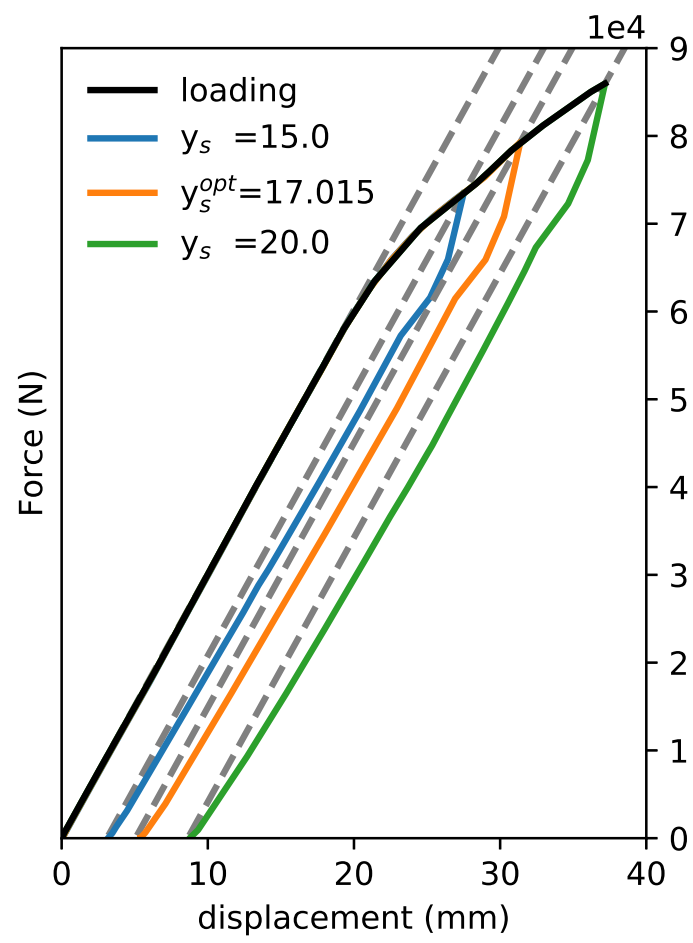

(b)

Fig. 17: T shaped beam. Force vs displacement diagram: numerical curves (solid lines) vs linear unloading path (grey dashed lines). (a) Reshaping configuration P1 (RS) and (b) Reshaping configuration P5 (RS).

Table 6: Calculation of the offset $\Delta y_{s}$ and error estimation for the applied stroke $y_{s}^{o p t}$.

\begin{tabular}{lrrc} 
Parameter / Configuration & P1 & P5 & \\
\hline Optimum stroke (RS) $y_{s}^{o p t, R S}$ & 12 & 17.015 & $\mathrm{~mm}$ \\
Optimum stroke (RSF) $y_{s}^{o p t, R S F}$ & 15.920 & 15.725 & $\mathrm{~mm}$ \\
Offset $\Delta y_{s}$ & -3.498 & 2.158 & $\mathrm{~mm}$ \\
Applied stroke $y_{s}^{o p t}$ & 12.422 & 17.883 & $\mathrm{~mm}$ \\
Relative Error $E_{r e l}$ & -3.5 & -5.1 & $\%$
\end{tabular}

differs to the actual optimum value $y_{s}^{o p t, R S}$ in less than $6 \%$. This gap is caused by the use of the linear unloading hypothesis to determine the offset. On the other hand, thanks to this hypothesis, the operator can retrieve nearly the optimal stroke in a single guess (no unloading).

The updated RSF curve after adding the offset $\Delta y_{s}$ is depicted in Figure ?? (in dashed lines). While $y_{s}^{\text {opt }}$ is determined in the synthetic reshaping diagram, when used in the real system (with residual stresses), the obtained remaining distortion $\delta\left(y_{s}^{o p t}\right)$ differs respect to the optimal value $\delta\left(y_{s}^{o p t, R S}\right)$, as shown in the detailed window for each Figure. To quantify the mismatch, the relative error $E_{r e l}$ respect to the distortion reduction is used. Thus, the distortion reduction is defined as the repair rate done after applying a selected value of stroke $\delta\left(y_{s}\right)$ respect to initial distortion in the current step $\left.\delta\right|_{y_{s}=0}$, see Eq.(??). Therefore, $E_{r e l}$ is obtained by normalizing the difference between the optimal and the obtained distortion reduction respect to the optimal distortion reduction, as described in Eq.(??). Note that the optimal distortion reduction corresponds to the maximum reparation rate achievable in the current reshaping step. As result, an error in the range of 10-15\% is obtained at the end of the process for configurations $\mathrm{P} 1$ and P5, respectively. All the values of the reshaping operation are summarized in Table ??.

distortion reduction $=\left(1-\frac{\delta\left(y_{s}\right)}{\left.\delta\right|_{y_{s}=0}}\right) \times 100(\%)$ 


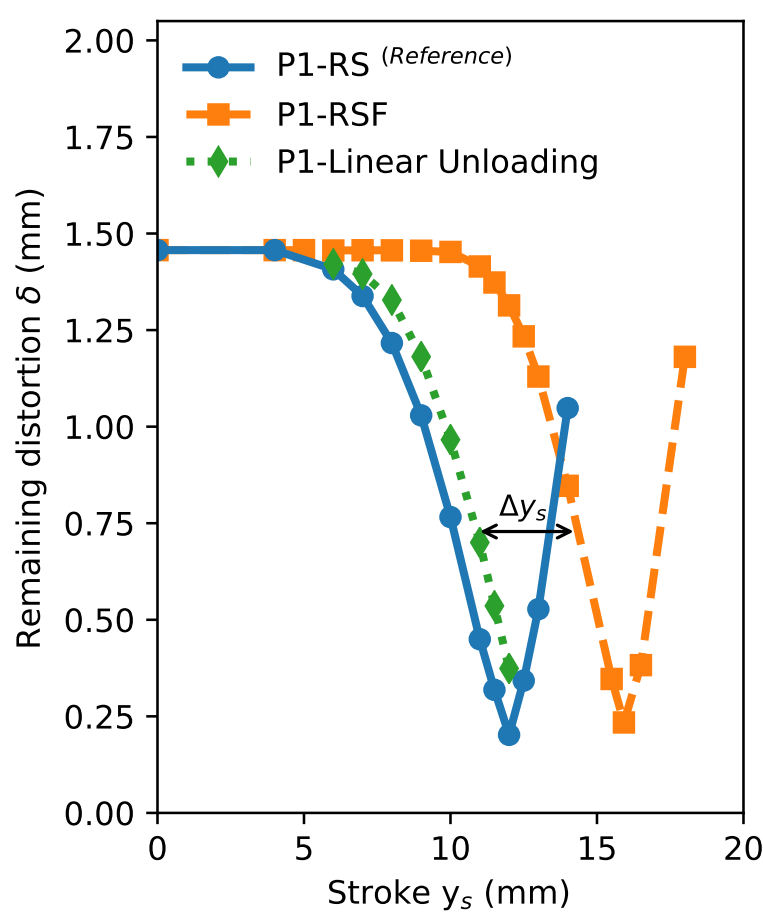

(a)

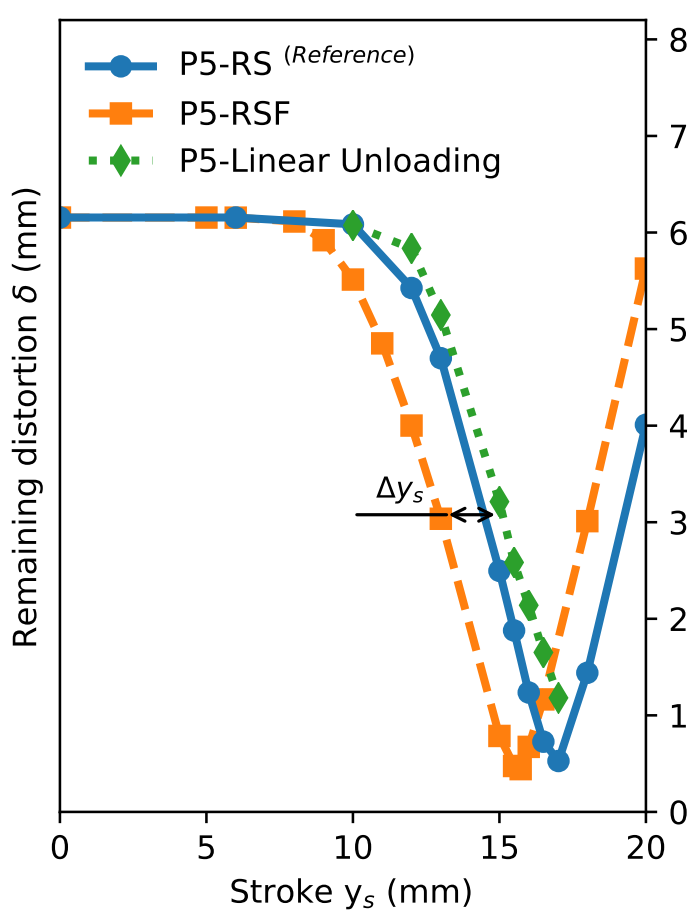

(b)

Fig. 18: T shaped beam. Remaining distortion vs Stroke diagram: Offset calculation. (a) Reshaping configuration P1 and (b) Reshaping configuration P5.

$E_{\text {rel }}=\left(1-\frac{\text { Obtained distortion reduction }}{\text { Optimal distortion reduction }}\right) \times 100(\%)$

To sum up, the advantage of using the proposed approach is that we can overcome the uncertainties related to the residual stresses present on the part, reduce the computational cost by neglecting the previous manufacturing steps and focus our efforts on the simulation of reshaping under the RSF hypothesis. Then, by using the LU path, it is not required to interrupt the repairing operation any more by performing any unnecessary unloading steps in order to calibrate the difference in response respect to the simulations obtained before. Now, only by tracking the force and displacement evolution during the process, it is possible to estimate an upper boundary of the remaining distortion $\delta$ as a function of the given stroke $y_{s}$. In other words, it is possible to reproduce an approximation of the reshaping diagrams with residual stresses obtained previously in Figure ?? to assist the operator in the task of repairing a part.

\section{Conclusions and perspectives}

In this paper, we have presented the reshaping diagrams as a tool for simulation assisted bending straightening. With this tool, it is possible to select in advance the stroke to apply in order to minimize distortion. Diagrams are generated in a two step procedure: $i$ ) off-line stage (numerical) and $i$ ) on-line stage (experimental). The off-line stage performs the reshaping simulation by considering a distorted part free of residual stresses and obtains the RSF-diagram. This simplification is able to describe the distortion evolution of the structure compared to the residual stress case with an offset. The on-line stage uses a linear unloading path to estimate the offset. This task is done by tracking the force and displacement while the reshaping operation in the real part is performed. Once the offset is known, the RSFdiagram is updated and an approximation to the optimum parameters is found. The relative error expected by using the reshaping diagram is around 10\%. From the computational point of view, the generation of the RSF-diagram is a very simple and inexpensive calculation that allows focusing on reshaping simulations only instead of the whole manufacturing chain, saving time 


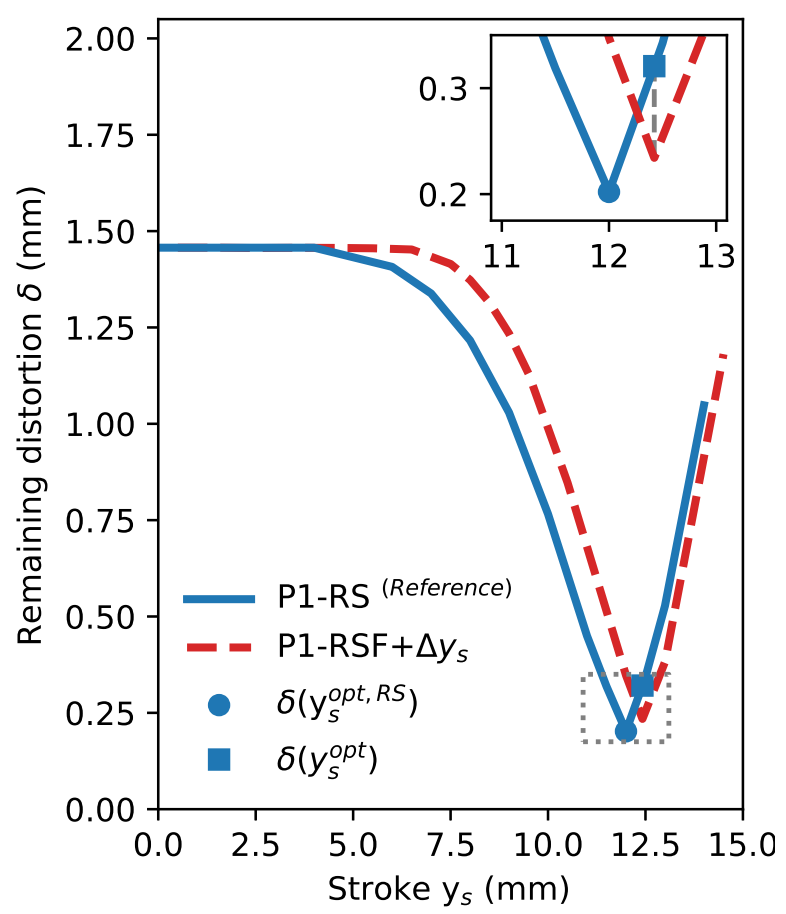

(a)

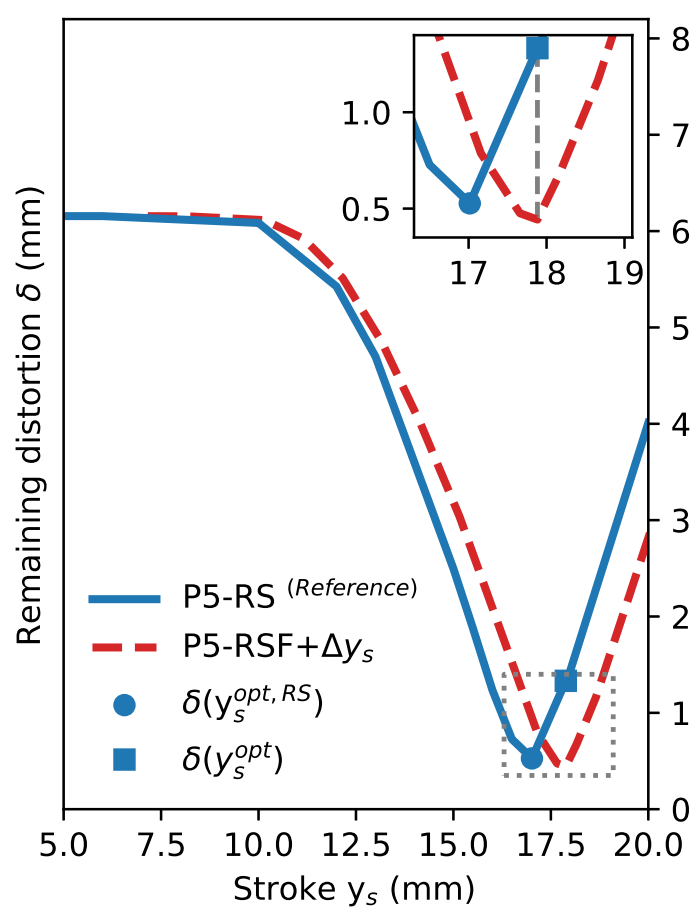

(b)

Fig. 19: T shaped beam. Reshaping diagram: optimal final distortion $\delta\left(y_{s}^{o p t, R S}\right)$ vs obtained final distortion $\delta\left(y_{s}^{o p t}\right)$. (a) Reshaping configuration P1 and (b) Reshaping configuration P5.

Table 7: Distortion reduction and error estimation after reshaping.

\begin{tabular}{lrrc} 
Parameter / Configuration & $\mathrm{P} 1$ & $\mathrm{P} 5$ & \\
\hline Initial distortion $\delta_{0}$ & 1.457 & 6.165 & $\mathrm{~mm}$ \\
Optimal final distortion (RS) $\delta\left(y_{s}^{\text {opt }, R S}\right)$ & 0.202 & 0.527 & $\mathrm{~mm}$ \\
Obtained final distortion (RS) $\delta\left(y_{s}^{\text {opt }}\right)$ & 0.321 & 1.332 & $\mathrm{~mm}$ \\
Optimal distortion reduction $(\mathrm{RS})$ & 86.1 & 91.5 & $\%$ \\
Obtained distortion reduction $(\mathrm{RS})$ & 78.0 & 78.4 & $\%$ \\
Relative Error $E_{\text {rel }}$ & 9.5 & 14.3 & $\%$
\end{tabular}

from modelling and simulation. Regarding the linear unloading path to determine the offset, it avoids interrupting the loading process during reshaping. Additionally, force and displacement are quantities that can be easily obtained in a daily industrial environment. As a limitation, it should be used only for thick-walled components.

The proposed approach reduces the gap between the theoretical outputs of numerical simulations and the practical needs at industrial level. The obtained results also opens the door to study reshaping under a Reduced Order Model (ROM) framework, because it is known how to parametrize shape [?], i.e the initial distorted geometry can be introduced as an extra parameter, while the task to parametrize $\mathrm{RS}$ is an open field yet. Future work will focus on the development of a ROM for the reshaping problem with the aim to explore in a virtual environment the influence of different parameters involved during the operation.

Acknowledgements This project has received funding from the European Union's Horizon 2020 research and innovation programme under the Marie Skłodowska-Curie grant agreement No 675919. The first author would like to thank Dominique Deloison for his invaluable help and comments during the development of this work. 


\section{Conflict of interest}

The authors declare that they have no conflict of inter-

est. 